\title{
Cdc42 localized in the CatSper signaling complex regulates cAMP-dependent pathways in mouse sperm
}

\author{
Guillermina M. Luque ${ }^{1}$ (D) | Xinran Xu $\mathbf{X u}^{2}$ | Ana Romarowski ${ }^{1,3}$ | María G. Gervasi ${ }^{3}$ (D) | \\ Gerardo Orta $^{4}$ | José L. De la Vega-Beltrán ${ }^{4}$ | Cintia Stival ${ }^{5}$ | Nicolás Gilio ${ }^{1}$ \\ Tomás Dalotto-Moreno $^{1}$ | Dario Krapf ${ }^{5}$ | Pablo E. Visconti ${ }^{3}$ | Diego Krapf ${ }^{2}$ | \\ Alberto Darszon $^{4}$ (I) | Mariano G. Buffone ${ }^{1}$ (iD
}

${ }^{1}$ Instituto de Biología y Medicina Experimental (IBYME-CONICET), Ciudad Autónoma de Buenos Aires, Argentina

${ }^{2}$ Department of Electrical and Computer Engineering, Colorado State University, Fort Collins, CO, USA

${ }^{3}$ Department of Veterinary and Animal Science, University of Massachusetts, Amherst, MA, USA

${ }^{4}$ Instituto de Biotecnología, UNAM, Cuernavaca, México

${ }^{5}$ Instituto de Biología Molecular y Celular de Rosario (CONICET-UNR), Rosario, Santa Fe, Argentina

\section{Correspondence}

Mariano G. Buffone, Instituto de Biología

y Medicina Experimental (IBYME-

CONICET), Vuelta de Obligado 2490

(1428), Ciudad Autónoma de Buenos Aires,

Argentina.

Email: mgbuffone@ibyme.conicet.gov.ar

\section{Funding information}

This work was supported by Agencia

Nacional de Promoción Científica y

Tecnológica (PICT 2015-2294, 2015-3164,

2017-3047, 2018-1988), the Williams and

Rene Baron Foundations, the Consejo

Nacional de Ciencia y Tecnología

(CONACyT - México Fronteras 71)

and Programa de Apoyo a Proyectos de

Investigación e Innovación Tecnológica

UNAM (PAPIIT/DGAPA IN205516). Part

of this work was performed, while $\mathrm{AD}$ was carrying out a sabbatical at the Instituto

Gulbenkian de Ciencia (IGC) supported by

UNAM/DGAPA and IGC

\begin{abstract}
Sperm acquire the ability to fertilize in a process called capacitation and undergo hyperactivation, a change in the motility pattern, which depends on $\mathrm{Ca}^{2+}$ transport by CatSper channels. CatSper is essential for fertilization and it is subjected to a complex regulation that is not fully understood. Here, we report that similar to CatSper, Cdc42 distribution in the principal piece is confined to four linear domains and this localization is disrupted in CatSper1-null sperm. Cdc42 inhibition impaired CatSper activity and other $\mathrm{Ca}^{2+}$-dependent downstream events resulting in a severe compromise of the sperm fertilizing potential. We also demonstrate that $\mathrm{Cdc} 42$ is essential for CatSper function by modulating cAMP production by soluble adenylate cyclase (sAC), providing a new regulatory mechanism for the stimulation of CatSper by the cAMP-dependent pathway. These results reveal a broad mechanistic insight into the regulation of $\mathrm{Ca}^{2+}$ in mammalian sperm, a matter of critical importance in male infertility as well as in contraception.
\end{abstract}

\section{K E Y W O R D S}

calcium, capacitation, hyperactivation, sperm, super-resolution

\footnotetext{
Abbreviations: 3D STORM, stochastic optical reconstruction microscopy; 8-Br-cAMP, 8-bromo-cyclic AMP; AKAP4, a kinase anchoring protein 4; BCECF AM, 2', 7'-Bis-(2-carboxyethyl)-5-(and-6)-carboxyfluorescein, acetoxymethyl ester; BSA, Bovine serum albumin; CAP, conditions that support sperm capacitation; CASA, computer-assisted semen analysis; CCCP, carbonyl cyanide m-chlorophenylhydrazone; db-cAMP, dibutyryl-cyclic AMP; $\mathrm{DiSC}_{3}(5), 3,3$-dipropylthiadicarbocyanine iodide; DVF, divalent cation-free solution; EGTA, ethylene glycol-bis (2-aminoethylether)-N,N,N,N'tetraacetic acid; Em, membrane potential; FSC-A, forward-scatter area; GDI, guanine nucleotide dissociation inhibitors; GEF, guanine nucleotide exchange factors; GLUT3, facilitative glucose transporter 3; GTP, guanosine 5'-triphosphate; HET, heterozygous; HRP, horseradish peroxidase-conjugated; IBMX, 3-isobutyl-1-methylxanthine; $I_{\text {CatSper }}$, CatSper monovalent currents; MFI, median fluorescence intensity; NC, conditions that do not support sperm capacitation; $\mathrm{NHA}, \mathrm{Na}^{+} / \mathrm{H}^{+}$antiporter; P-CaMKII, phosphorylated $\mathrm{Ca}^{2+} /$ calmodulin-dependent protein kinase II; $\mathrm{pH}_{\mathrm{i}}$, intracellular $\mathrm{pH}$; PI, propidium iodide; pPKAs, phospho PKA substrates; pY, tyrosine phosphorylation; sAC, soluble adenylate cyclase; sNHE, sperm-specific $\mathrm{Na}^{+} / \mathrm{H}^{+}$exchanger; TYH, ToyodaYokoyama-Hosi; $\left[\mathrm{Ca}^{2+}\right]_{\mathrm{i}}$, intracellular $\mathrm{Ca}^{2+}$ concentration.
} 


\section{1 | INTRODUCTION}

The Rho-family of small GTPase proteins (Cdc42, Rac, and Rho) govern a variety of important cellular functions in all tissues. ${ }^{1}$ Rho GTPases act by a tightly regulated switch between their inactive GDP-bound and their active GTP-bound state. In particular, Cdc42 is now known to influence several signaling events and cellular processes in different organisms, from yeast to mammals. The presence of $\mathrm{Cdc} 42$ and other small GTPases together with some of their downstream effectors have been described in mammalian sperm $^{2-6}$ but their role has not been clearly elucidated. One of the reasons for this lack of knowledge is that the global targeted deletion of these proteins results in embryonic lethality due to their critical function in several tissues. ${ }^{7}$ In the case of $\mathrm{Cdc} 42$, it is essential for the blood-testis barrier function ${ }^{8}$ and is largely associated with spermatocytes and Sertoli cells. For this reason, the germ-cell-specific targeted deletion will result in abnormal spermatogenesis. ${ }^{9}$ Altogether, these limitations have impeded to study Cdc42 function in mature sperm other than with pharmacological approaches.

Sperm acquire the ability to fertilize in the female genital tract in a process called capacitation. ${ }^{10,11}$ From a molecular point of view, bicarbonate $\left(\mathrm{HCO}_{3}{ }^{-}\right)$and $\mathrm{Ca}^{2+}$ stimulation of the soluble adenylyl cyclase (sAC; aka ADCY10) lead to an increase in cAMP, PKA activity, and complex regulation of tyrosine phosphorylation (pY) of sperm proteins. ${ }^{12-14}$ Activation of cAMP-dependent signaling pathways leads to an increase in intracellular $\mathrm{Ca}^{2+15-17}$ that triggers a change in the motility pattern called hyperactivation, which is critical for fertilization. ${ }^{18}$ This high-amplitude, asymmetric, whiplike beating pattern of the flagellum enables sperm to migrate through the oviduct and to overcome the oocyte surrounding layers. ${ }^{19,20}$

The $\mathrm{Ca}^{2+}$ uptake required for hyperactivation occurs through the sperm-specific CatSper channel complex localized in the plasma membrane of the principal piece of mature sperm. ${ }^{21-23}$ This complex comprises four homologous $\alpha$ subunits (CatSper 1-4) ${ }^{24,25}$ and auxiliary subunits: CatSper $\beta$, CatSper $\gamma$, CatSper $\delta,{ }^{26-28}$ and the more recently described CatSper $\varepsilon$, CatSper $\zeta$, and EFCAB9.$^{29,30}$ Mice lacking any of the CatSper1-4 genes ${ }^{21,31,32}$ as well as human males with CatSper loss of function mutations ${ }^{33-35}$ are infertile and their sperm unable to hyperactivate.

The CatSper channel is probably the most complex of all ion channels characterized at the moment. It provides the fine-tuning for $\mathrm{Ca}^{2+}$ homeostasis required for changes in motility that sperm experience during their transit to the fertilization site. In addition, CatSper channels are subjected to a sophisticated regulation that differs according to the species. For example, exogenous compounds such as progesterone and prostaglandins produce a robust channel activation in human but not mouse sperm $^{36,37}$ mediated by the degradation of lipid endocannabinoid 2-arachidonoylglycerol (2AG). ${ }^{38}$ In contrast, both mouse and human CatSper channels are activated by intracellular alkalinization. ${ }^{39,40}$ This indicates species-specific adaptations of sperm to adjust to a distinct set of activators within the female reproductive tract. ${ }^{41}$

The complex structural organization of CatSper has impeded the heterologous reconstitution of the channel to study its regulation. It was recently reported that CatSper proteins form a unique pattern of four linear domains in the plasma membrane along the principal piece of the flagellum. ${ }^{29,30,42}$ This segregated localization of proteins may provide the structural basis for the selective activation of CatSper and the development of hyperactivated motility. In addition, other signaling molecules such as Caveolin-1, phosphorylated $\mathrm{Ca}^{2+} /$ calmodulin-dependent protein kinase II (P-CaMKII), calcineurin (PPP3C aka PP2B), and EFCAB9 colocalize with CatSper displaying a similar spatial distribution along the principal piece in mouse sperm. ${ }^{30,42}$ Only CatSper has been shown to be critical for the organization of these nanodomains since CatSpernull mice do not display the four-column organization of these signaling molecules. ${ }^{42}$ It is anticipated that the proteins included in the signaling complex with CatSper may constitute an important point of regulation for this channel. However, with the exception of EFCAB9 that provides partial $\mathrm{pH}$ and $\mathrm{Ca}^{2+}$ sensitivity, ${ }^{30}$ no report has demonstrated regulatory functions on CatSper activity.

In this report, we found an unexpected new role for the small GTPase Cdc42 in mouse sperm. Using a multidisciplinary approach, we provide evidence that $\mathrm{Cdc} 42$ is a new regulatory protein that localizes in the CatSper nanodomains complex and has a critical function on the activity of this channel by modulating cAMP production through sAC. As such, Cdc42 is essential for hyperactivation and ultimately, for fertilization.

\section{MATERIALS AND METHODS}

\section{1 | Reagents}

Chemicals were obtained from the following sources: bovine serum albumin (BSA) A7906, 3-isobutyl-1-methylxanthine (IBMX), dibutyryl-cyclic AMP (db-cAMP), Mibefradil, NNC55-0396, anti- $\beta$-tubulin T4026, protease inhibitor cocktail P8340, carbonyl cyanide m-chlorophenylhydrazone (CCCP), guanosine 5'-triphosphate sodium salt hydrate (GTP) G8877, and ethylene glycol-bis (2-aminoethylether)$\mathrm{N}, \mathrm{N}, \mathrm{N}, \mathrm{N}$ 'tetraacetic acid (EGTA) were purchased from Sigma-Aldrich (St. Louis, MO, USA). Anti-phosphotyrosine (anti-pY) clone 4G10 and anti-facilitative glucose transporter 3 (anti-GLUT3) AB1344 were purchased from Millipore 
(Temecula, CA, USA); anti-phospho PKA substrates (antipPKAs) clone 100G7E and anti-phospho CaMKII (Thr286) 3361 from Cell Signaling Technology (Danvers, MA, USA); horseradish peroxidase-conjugated (HRP) anti-rabbit IgG and HRP anti-mouse IgG from Vector Laboratories (Burlingame, CA, USA). 2',7'-Bis-(2-Carboxyethyl)-5-(a nd-6)-Carboxyfluorescein, acetoxymethyl ester (BCECF AM), 8-Bromo-cyclic AMP (8-Br-cAMP, sodium salt), KT5720, CASIN, MLS-573151, ML-141, and ZCL278 were purchased from Cayman Chemicals (Ann Arbor, MI, USA). Secramine A was kindly provided from the Kirchhausen Lab (Harvard Medical School, MA, USA). Fluo-4 AM, 3,3-dipropylthiadicarbocyanine iodide $\left(\operatorname{DiSC}_{3}(5)\right)$, pluronic acid, F(ab')2-goat anti-rabbit IgG-Alexa Fluor 647 (A-21246), and chicken anti-mouse IgG-Alexa Fluor 647 (A-21463) were purchased from Invitrogen, Thermo Fisher Scientific (Waltham, MA, USA); while propidium iodide (PI), antiCdc42 (B-8) sc-8401, Cdc42 (B-8) Blocking Peptide sc-8401 $\mathrm{P}$, anti-Cdc42 sc-87, and anti-Actin sc-1616-R were purchased from Santa Cruz Biotechnology (Dallas, TX, USA). Anti-AKAP82 611564 was purchased from BD Biosciences (Franklin Lakes, NJ, USA). Anti- $\beta$-tubulin E7 was purchased from Developmental Studies Hybridoma Bank (DBHS) University of Iowa; E7 was deposited to the DSHB by Michael Klymkowsky. All other chemicals were of reagent grade. Fluo-4 AM, $\mathrm{DiSC}_{3}(5)$, pluronic acid, IBMX, KT5720, CCCP, CASIN, MLS-573151, ML-141, ZCL278, and Secramine A were dissolved in DMSO; EGTA, 8-Br-cAMP, db-cAMP, Mibefradil, NNC55-0396, and PI were dissolved in hexa-distilled water.

\section{2 | Animals}

Hybrid F1 (C57BL/6 male $\mathrm{x}$ Balb/C female) and CD-1 mature (10-12 weeks old) male mice were used. CatSper1 $\mathrm{KO}^{21}$ mice and their corresponding heterozygous (HET) siblings (C57BL/6) reside at the University of Massachusetts. ${ }^{43}$ CatSper1-null mice were euthanized in accordance with the Animal Care and Use Committee (IACUC) guidelines of UMass-Amherst (protocol \#20160026). In all cases, mice were housed in groups of 4 or 5 in a temperature-controlled room $\left(23^{\circ} \mathrm{C}\right)$ with lights on at 07:00 hours and off at 19:00 hours, and had free access to tap water and laboratory chow. All experimental procedures were carried according to the guidelines of the institutional animal care and were reviewed and approved by the Ethical Committees of the Instituto de Biología y Medicina Experimental, Buenos Aires, and of the Instituto de Biotecnología, Cuernavaca. Experiments were performed in strict accordance with the Guide for Care and Use of Laboratory Animals approved by the National Institutes of Health (NIH).

\subsection{Sperm medium}

The non-capacitating medium used in this study was a modified Toyoda-Yokoyama-Hosi (modified TYH) containing $119.3 \mathrm{mM} \mathrm{NaCl}, 4.7 \mathrm{mM} \mathrm{KCl}, 1.71 \mathrm{mM} \mathrm{CaCl}{ }_{2} .2 \mathrm{H}_{2} \mathrm{O}$, $1.2 \mathrm{mM} \mathrm{KH}_{2} \mathrm{PO}_{4}, 1.2 \mathrm{mM} \mathrm{MgSO}{ }_{4} .7 \mathrm{H}_{2} \mathrm{O}, 0.51 \mathrm{mM}$ sodium pyruvate, $5.56 \mathrm{mM}$ glucose, $20 \mathrm{mM}$ HEPES, and $10 \mu \mathrm{g} / \mathrm{mL}$ of gentamicin (NC medium). For capacitating conditions, $15 \mathrm{mM} \mathrm{NaHCO}_{3}$ and $5 \mathrm{mg} / \mathrm{mL}$ of BSA were added (CAP medium). In all cases, $\mathrm{pH}$ was adjusted to 7.4 with $\mathrm{NaOH}$. In in vitro fertilization assays, $20 \mathrm{mM}$ HEPES was omitted, while $25 \mathrm{mM} \mathrm{NaHCO}$ and $4 \mathrm{mg} / \mathrm{mL}$ of BSA were added (TYH IVF).

\subsection{Sperm capacitation}

Animals were euthanized and cauda epididymal mouse sperm were collected. Both cauda epididymis were placed in $1 \mathrm{~mL}$ of non-capacitating modified TYH medium (NC: without BSA and $\mathrm{NaHCO}_{3}$ ) in the presence or absence of $\mathrm{Ca}^{2+}$ as described for each experiment. After 15 minutes of incubation at $37^{\circ} \mathrm{C}$ (swim-out), epididymis was removed and sperm were resuspended to a final maximum concentration of $1 \times 10^{7}$ cells $/ \mathrm{mL}$ on $100 \mu \mathrm{L}$ of the appropriate medium, depending on the experiment performed. A 10 minutes pre-incubation in $100 \mu \mathrm{L}$ of $\mathrm{NC}$ medium containing Cdc42 inhibitors (MLS573151, CASIN or Secramine A), CatSper inhibitors (30 $\mu \mathrm{M}$ Mibefradil or $10 \mu \mathrm{M}$ NNC55-0396) or PKA inhibitor (30 $\mu \mathrm{M}$ KT-5720) was conducted when required. An equal volume $(100 \mu \mathrm{L})$ of $\mathrm{NC}$ or twofold concentrated capacitating medium (CAP 2X: $30 \mathrm{mM} \mathrm{NaHCO}$ and $10 \mathrm{mg} / \mathrm{mL}$ of BSA) containing the appropriate inhibitors was added. When necessary, $1 \mathrm{mM}$ 8-Br-cAMP or db-cAMP (permeable cAMP analogs) in combination with $0.2 \mathrm{mM}$ IBMX (phosphodiesterase inhibitor) was also added. Finally, sperm were incubated for 90 minutes at $37^{\circ} \mathrm{C}$.

\section{5 | Three-dimensional stochastic optical reconstruction microscopy}

After swim-out, cells were washed twice with $\mathrm{NC}$ medium by centrifugation (5 minutes at $400 \mathrm{~g}$ ) and finally resuspended in $\mathrm{NC}$ medium. Sperm were seeded in poly-L-lysine coated coverslips (Corning \#1.5), air-dried for 10 minutes, fixed with $4 \%$ fresh paraformaldehyde in PBS for 10 minutes at room temperature, and followed by three washes with PBS (5 minutes at $400 \mathrm{~g}$ ). Cells were then permeabilized with $0.5 \%$ Triton X-100 in PBS for 5 minutes at room temperature and washed three times with PBS (5 minutes at $400 \mathrm{~g}$ ). Samples were blocked with 3\% BSA/PBS for 1 hour at room temperature and then incubated overnight at $4^{\circ} \mathrm{C}$ with primary antibody in a humidified 
chamber: anti-Cdc42 (1:100), anti- $\beta$-tubulin E7 (1:100), antiAKAP4 (1:300), anti-GLUT3 (1:100) or anti-P-CaMKII (1:50), all diluted in $1 \% \mathrm{BSA} / \mathrm{PBS}$. Cells were washed three times with T-PBS (5 minutes at $400 \mathrm{~g}$ ), and further incubated with Alexa Fluor 647-conjugated anti-mouse (1:500) or rabbit (1:1000) secondary antibody diluted in 1\% BSA/PBS for 1 hour at room temperature. Sperm were then washed with T-PBS twice and PBS once for 5 minutes each, and immediately mounted in STORM imaging buffer (50 mM Tris- $\mathrm{HCl}$ pH 8, $10 \mathrm{mM} \mathrm{NaCl}$, $0.56 \mathrm{mg} / \mathrm{mL}$ of glucose oxidase, $34 \mu \mathrm{g} / \mathrm{mL}$ of catalase, $10 \%$ glucose, and $1 \% \beta$-mercaptoethanol). Nonspecific staining was determined by incubating the sperm in the absence of primary antibody. Images were acquired using Andor iQ 2.3 software in a custom-built microscope equipped with an Olympus Plan Apo 100x NA 1.45 objective and a CRISP ASI autofocus system. $^{44,45}$ Alexa Fluor 647 was excited with a $642 \mathrm{~nm}$ laser (DL640-150-O, CrystaLaser, Reno, NV) under continuous illumination. Initially, the photoswitching rate was sufficient to provide a substantial fluorophore density. However, as fluorophores irreversibly photo-bleached, a $405 \mathrm{~nm}$ laser was introduced to enhance photoswitching. The intensity of the $405 \mathrm{~nm}$ laser was adjusted in the range of $0.01-0.5 \mathrm{~mW}$ to maintain an appropriate density of active fluorophores. Axial localization was achieved via astigmatism using a MicAO 3DSR adaptive optics system (Imagine Optic, Orsay, France) that allows both the correction of spherical aberrations and the introduction of astigmatism. ${ }^{46}$ Axial localization with adaptive optics enabled 3D reconstruction over a thickness of $1 \mu \mathrm{m} .{ }^{47} \mathrm{~A}$ calibration curve for axial localization was generated with $100 \mathrm{~nm}$ TetraSpeck microspheres (Invitrogen) immobilized on a coverslip. ${ }^{48}$ The images were acquired in a water-cooled, backilluminated EMCCD camera (Andor iXon DU-888) operated at $-85^{\circ} \mathrm{C}$ at a rate of 23 frames/sec. A total of 50,000 frames were collected to generate each super-resolution image. Singlemolecule localization, drift correction using image crosscorrelation, and reconstruction were performed with Thunder STORM. $^{49}$

To find the molecular radial distributions, we selected regions of interest of the flagellum that were found to lie in a straight line. The center of the flagellar cross-section was first found by the Gaussian fitting of the localization histograms along $\mathrm{x}$ and $\mathrm{y}$. The coordinates of the localized molecules were then transformed into cylindrical coordinates to obtain the radial position $r$ and azimuthal angle $\Theta .^{47,50}$

\subsection{Determination of $\left[\mathrm{Ca}^{2+}\right]_{\mathrm{i}}$ and $\mathrm{pH}_{\mathrm{i}}$ by flow cytometry}

Sperm intracellular $\mathrm{Ca}^{2+}$ concentration $\left(\left[\mathrm{Ca}^{2+}\right]_{\mathrm{i}}\right)$ and intracellular $\mathrm{pH}\left(\mathrm{pH}_{\mathrm{i}}\right)$ changes were assessed using Fluo-4 AM and BCECF AM, respectively, as previously described. ${ }^{17}$ After incubation in the appropriate medium, samples were centrifuged at $400 \mathrm{~g}$ for 4 minutes at room temperature and resuspended in $200 \mu \mathrm{L}$ of $\mathrm{NC}$ medium containing either $1 \mu \mathrm{M}$ Fluo-4 AM and $0.02 \%$ pluronic acid or $0.5 \mu \mathrm{M}$ BCECF AM for 20 minutes at $37^{\circ} \mathrm{C}$. Samples were washed again and resuspended in $50 \mu \mathrm{L}$ of NC medium. Before collecting data, $2 \mathrm{ng} /$ $\mu \mathrm{L}$ of PI was added to monitor viability. Data were recorded as individual cellular events using a BD FACSCanto II TM cytometer (Biosciences; Becton, Dickinson and Company). Side-scatter area (SSC-A) and forward-scatter area (FSC-A) data were collected from 20000 events per sample in order to define sperm population as previously described. ${ }^{51}$ In all cases, doublet exclusion was performed analyzing twodimensional dot plot FSC-A vs forward-scatter height (FSC$\mathrm{H})$. Positive cells for Fluo-4 AM were collected using the filter for Fluorescein isothiocyanate (FITC; 530/30), and for PI the filter for Peridinin chlorophyll protein complex (PerCP; 670LP). The two indicators had minimal emission overlap, but still compensation was performed. Data were analyzed using FlowJo software (V10.0.7).

\subsection{Cdc42 activation assay}

To specifically detect the GTP-loaded form of Cdc42, the Cd42 G-LISA kit (Cytoskeleton Inc, Denver, CO, USA) was used according to the manufacturer's instructions. Briefly, $50 \mu \mathrm{L}$ of duplicate samples of lysates from sperm incubated for 90 minutes under capacitating conditions (CAP) in the absence (DMSO) or presence of Cdc42 inhibitor $(20 \mu \mathrm{M}$ MLS-573151) at a concentration between 0.65 and $0.95 \mathrm{mg}$ protein $/ \mathrm{mL}$ were added into each well. Plain lysis buffer and a standard of constitutively active purified GTP-bound Cdc42 protein were added to duplicate wells as a blank and positive control, respectively. After binding, anti-Cdc42 primary antibody was added to each well followed by a secondary antibody labeled with HRP, which was developed by adding HRP reagent. Each well was read at OD $492 \mathrm{~nm}$ on a 96-well plate spectrophotometer. Lysis buffer background was subtracted and results were normalized to protein concentration.

\section{8 | Membrane potential assay in cell populations}

The procedure was performed as previously described. ${ }^{52}$ Sperm obtained from swim-out were diluted in NC or CAP medium and loaded with $1 \mu \mathrm{M}$ of the membrane-potential-sensitive dye $\mathrm{DiSC}_{3}(5)$ for 5 minutes. Mitochondrial membrane potential was dissipated incubating sperm for 2 minutes with $500 \mathrm{nM} \mathrm{CCCP.}$ After this, $1 \mathrm{~mL}$ of the sperm suspension was transferred to a stirred cuvette at $37^{\circ} \mathrm{C}$ and fluorescence monitored with an Ocean Optics USB4000 spectrofluorometer operated by Spectra Suite (Ocean Optics, Inc, USA) at 620/670 nm excitation/emission 
wavelength pair. ${ }^{53}$ Cell hyperpolarization decreases the dye fluorescence. Recordings were initiated after reaching steady-state fluorescence (1-3 minutes) and calibration was performed at the end of each measure by adding $1 \mu \mathrm{M}$ valinomycin and sequential additions of $\mathrm{KCl}$ as previously described. ${ }^{52}$ Sperm membrane potential (Em) was obtained from the initial fluorescence (measured as Arbitrary Fluorescence Units) by linearly interpolating it in the theoretical Em values for the calibration curve against arbitrary fluorescence units of each trace. This internal calibration for each determination compensates for variables that influence the absolute fluorescence values.

On one hand, CatSper activity was assessed by measuring Em in TYH medium after adding EGTA ( $3.5 \mathrm{mM}$ final, $\mathrm{pH}$ adjusted with $\mathrm{NaOH}$ to $\sim 10$ so that media $\mathrm{pH}$ does not change upon $\mathrm{H}^{+}$release in exchange for $\mathrm{Ca}^{2+}$ ), which allows $\mathrm{Na}^{+}$influx through CatSper, depolarizing non-capacitated sperm. The magnitude of the depolarization caused by $\mathrm{Na}^{+}$ influx correlates with the extent of CatSper opening. Briefly, after sperm treatment (capacitation in presence or absence of Cdc42 inhibitors), cells were collected by centrifugation (400 $\mathrm{g}, 3$ minutes) and concentration adjusted to $2 \times 10^{6}$ sperm $/ \mathrm{mL}$ with NC medium with the addition of the corresponding inhibitor or not. Then, sperm were loaded with $1 \mu \mathrm{M}$ of $\operatorname{DiSC}_{3}(5)$. Results were represented as $\Delta$ Fluorescence, the difference between $\operatorname{DiSC}_{3}(5)$ fluorescence after $3.5 \mathrm{mM}$ EGTA addition and before (resting Em) ${ }^{54}$ In every case, the fluorescence is calculated once is stabilized for at least one minute. In these experiments, calibration curves were performed to confirm that sperm were viable. Em values were not calculated from these calibrations.

On the other hand, non-capacitated sperm were loaded with $1 \mu \mathrm{M}$ of $\operatorname{DiSC}_{3}(5)$ and then exposed to $10 \mathrm{mM} \mathrm{NH}_{4} \mathrm{Cl}$, with prior addition of $\mathrm{Cdc} 42$ inhibitor or not. Alkalinization allows CatSper activation, which results in $\mathrm{Ca}^{2+}$ influx depolarizing the cell. Again, the magnitude of the depolarization correlates with the extent of CatSper opening. Results were represented as $\Delta \mathrm{Em}$, the difference between $\mathrm{Em}$ after $10 \mathrm{mM}$ $\mathrm{NH}_{4} \mathrm{Cl}$ addition and before (Em with or without $\mathrm{Cdc} 42$ inhibitor). In every case the fluorescence is calculated once is stabilized for at least one minute.

\section{9 | Electrophysiology}

Cauda epididymal sperm obtained from swim-out were resuspended in HS medium containing: $\mathrm{NaCl} 135 \mathrm{mM}, \mathrm{KCl} 5 \mathrm{mM}$, $\mathrm{CaCl}_{2} 2 \mathrm{mM}, \mathrm{MgSO}_{4} 1 \mathrm{mM}$, lactic acid $10 \mathrm{mM}$, pyruvic acid $1 \mathrm{mM}$, glucose $5 \mathrm{mM}$, HEPES $20 \mathrm{mM}$, and $\mathrm{pH} 7.4(\mathrm{NaOH})$. One hundred microliter aliquots of the cell suspension were dispensed into a recording chamber ( $1 \mathrm{~mL}$ total volume) and subjected to electrophysiological recording. Whole-cell macroscopic CatSper monovalent currents $\left(I_{\text {CatSper }}\right)$ were obtained by patch-clamping the sperm cytoplasmic droplet in cauda epididymal sperm and were analyzed as reported previously. ${ }^{39,54}$ Seals between the patch pipette and the cytoplasmic droplet in sperm were formed in HS bath solution (baseline current). After establishing the whole-cell configuration, the bath solution was changed for divalent cation-free solution (DVF) which is best to measure CatSper-dependent currents. The DVF bath solution contained: Na-gluconate $150 \mathrm{mM}$, $\mathrm{Na}_{2}$ EDTA 2 mM, EGTA 2 mM, HEPES 20 mM, pH 7.4, while the pipette solution contained: Met-Cs $135 \mathrm{mM}, \mathrm{CsCl}$ $5 \mathrm{mM}$, Na-ATP $5 \mathrm{mM}$, EGTA $10 \mathrm{mM}$, HEPES $10 \mathrm{mM}$, pH 7.0. When required, $0.4 \mathrm{mM}$ of GTP was added to the patchclamp pipette solution. The osmolarity of all solutions was adjusted with dextrose. All recordings were performed using a patch-clamp amplifier (Axopatch 200 Molecular Devices) at room temperature $\left(22^{\circ} \mathrm{C}\right)$. Pulse protocols and data capture were performed using pCLAMP6 software (Molecular Devices); data analysis was carried out with Clampfit 10.6 (Molecular Devices), Origin 7.5 (Microcal Software), and Sigma Plot 10 (SYSTAT Software). Current records, unless indicated otherwise, were acquired at $20-100 \mathrm{kHz}$ and filtered at $5-10 \mathrm{kHz}$ (low-pass Bessel filter) using a computer attached to a Digidata 1200 (Molecular Devices). Patch pipettes were pulled from borosilicate glass (Kimble Queretaro)

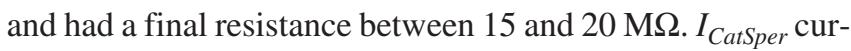
rents were evoked employing a conventional voltage-ramp protocol from $-80 \mathrm{mV}$ to $+80 \mathrm{mV}$, with a duration of $750 \mathrm{~ms}$ from a holding potential of $0 \mathrm{mV}$. In all cases, the addition of Cdc42 inhibitors was recorded until reaching a stable-state effect (3-5 minutes).

\subsection{0 | Extraction of sperm proteins and western blotting}

After incubation in the appropriate medium, sperm were washed by centrifugation ( 5 minutes at $400 \mathrm{~g}$ ), resuspended in sample buffer without reducing agents $(62.5 \mathrm{mM}$ Tris$\mathrm{HCl} \mathrm{pH} 6.8,2 \%$ SDS, $10 \%$ glycerol), and boiled for $5 \mathrm{~min}-$ utes. After centrifugation for 5 minutes at $13000 \mathrm{~g}, 5 \%$ $\beta$-mercaptoethanol and $0.0005 \%$ bromophenol blue were added to the supernatants then boiled again for 5 minutes. Protein extracts equivalent to $2-4 \times 10^{6}$ sperm per lane were separated by SDS-PAGE in gels containing $10 \%$ polyacrylamide and transferred onto nitrocellulose membranes. For Cdc42 detection, after being washed sperm were incubated in RIPA buffer (50 mM Tris, $150 \mathrm{mM} \mathrm{NaCl}, 0.5 \%$ sodium deoxycholate, $1 \%$ NP- $40,0.1 \%$ SDS) with protease inhibitor cocktail $1 \mathrm{X}$ for 20 minutes on ice, and proteins from 10 to $14 \times 10^{6}$ sperm per lane were separated by SDS-PAGE in $15 \%$ polyacrylamide gels. In all cases, blots were blocked in 5\% nonfat dry milk in PBS containing $0.1 \%$ Tween 20 (T-PBS) for 1 hour at room temperature. An overnight incubation at $4^{\circ} \mathrm{C}$ with the primary antibody anti-Cdc 42 was 
required, while 1 hour incubation at room temperature with the other primary antibodies was sufficient. Antibodies were diluted in $2 \%$ nonfat dry milk in T-PBS as follows: 1:500 for anti-Cdc42 (Santa Cruz sc-8401); 1:750 for antiCdc42 (Santa Cruz sc-87); 1:3000 for anti-pPKAs, anti-pY, and anti- $\beta$-tubulin T4026. The corresponding secondary antibodies were incubated for 1 hour at room temperature, diluted in 2\% nonfat dry milk in T-PBS as follows: 1:3000 for HRP anti-rabbit and 1:3000 for HRP anti-mouse. In all cases, the reactive bands were visualized using a chemiluminescence detection solution consisting of $100 \mathrm{mM}$ Tris$\mathrm{HCl}$ buffer $\mathrm{pH} 8,205 \mu \mathrm{M}$ coumaric acid, $1.3 \mathrm{mM}$ luminol, $0.01 \% \mathrm{H}_{2} \mathrm{O}_{2}$, and were exposed for different time periods to CL-XPosure film (Thermo Scientific). In all experiments, molecular masses were expressed in kilodalton (kDa). ImageJ 1.48k (National Institute of Health, USA) was used for the analysis of the western blot images following the specifications of ImageJ User Guide, IJ 1.46r. The optical density of all bands was quantified and normalized, first to the $\beta$-tubulin or actin band and then to the capacitating condition of each experiment.

\subsection{1 | Immunofluorescence}

To perform immunofluorescence on mouse sperm, a previously described method was used. ${ }^{47}$ Briefly, mouse sperm were washed twice by centrifugation for 5 minutes at $400 \mathrm{~g}$, resuspended in PBS containing 4\% paraformaldehyde (Electron Microscopy Sciences, Hatfield, PA, USA) for 10 minutes at room temperature. After washing twice for 5 minutes in PBS, sperm were placed onto glass slides. Sperm were airdried and then permeabilized with $0.5 \%$ Triton X-100 in PBS for 5 minutes. The slides were washed twice for 5 minutes in T-PBS and blocked in 3\% BSA in PBS for 1 hour at room temperature, then were incubated with primary antibody (1:100 mouse monoclonal anti-Cdc42 (B-8), sc-8401) diluted in PBS containing $1 \% \mathrm{BSA}$ overnight at $4^{\circ} \mathrm{C}$. Following a washing step (three times, 5 minutes in T-PBS), slides were incubated with Alexa Fluor 488-conjugated goat anti-mouse IgG (Invitrogen, A11001) diluted 1:500 in PBS containing $1 \%$ BSA for 1 hour at room temperature, washed again for three times in T-PBS, and mounted using VECTASHIELD mounting media (H-1000, Vector Labs, Burlingame, CA, USA). Nonspecific staining was determined by incubating the sperm with blocked antibody. To this end, $1 \mu \mathrm{g}$ of the primary antibody (anti-Cdc42 (B-8), sc-8401) was pre-incubated with $20 \mu \mathrm{g}$ of blocking peptide (Cdc42 (B-8), sc-8401 P) in PBS, for 2 hours at room temperature with agitation. Therefore, the blocking peptide was 20 times more concentrated than the primary antibody. Slides were examined using an inverted fluorescence microscope (Nikon TE300) and images were captured at $60 \times$ magnification (Plan Apo, NA1.4(oil)), with an sCMOS camera (Andor, Zyla).

\subsection{2 | Determination of cAMP levels}

Intracellular sperm cAMP concentrations were determined using a cAMP select ELISA kit (Cayman Chemicals, Ann Arbor, MI, USA). After the incubation of sperm in the appropriate conditions, reactions were stopped with $0.1 \mathrm{M} \mathrm{HCl}$, and cells were lysed with Triton $1 \%$. This ELISA-based assay has an increased sensitivity that ranges from 0.02 to 40 pmol of cAMP. A standard curve was run for each assay and the unknown cAMP concentrations were obtained by interpolation as recommended by the manufacturer.

\subsection{Sperm motility analysis}

After incubation in the appropriate medium, sperm suspensions were loaded on a $100 \mu \mathrm{m}$ chamber slide (Leja Slide, Spectrum Technologies) and placed on a microscope (Nikon Eclipse E200) stage at $37^{\circ} \mathrm{C}$ coupled with a BASLER acA780-75gc camera. Sperm movements were examined using computer-assisted semen analysis (CASA) system (Sperm Class Analyzer: SCA evolution, Microptic). Parameters used were as follows: 30 frames acquired, frame rate of $60 \mathrm{~Hz}$, and cell size of $30-170 \mu \mathrm{m}^{2}$. At least 20 microscopy fields corresponding to a minimum of 200 sperm were analyzed in each experiment. The following parameters were measured: mean path velocity (VAP), curvilinear velocity (VCL), straight-line velocity (VSL), linearity (LIN), amplitude of lateral head displacement (ALH), and straightness (STR). Sperm were considered hyperactivated when presenting VCL $\geq 271 \mu \mathrm{m} / \mathrm{sec}, \mathrm{LIN}<50 \%$, and ALH $\geq 3.5 \mu \mathrm{m}$.

\subsection{In vitro fertilization assay}

Eight to 12-week-old F1 female mice were superovulated using equine chorionic gonadotropin (5 IU, PMSG; Syntex) at 19:30, followed 48 hours later by human chorionic gonadotropin (5 IU, hCG; OVUSYN, Syntex) intraperitoneal injection. Cumulus-oocyte complexes (COC) were collected from oviducts 12-13 hours after hCG administration, placed in TYH IVF medium (containing $25 \mathrm{mM} \mathrm{NaHCO}$ and $4 \mathrm{mg} / \mathrm{mL}$ of BSA, with no HEPES addition), and inseminated in vitro with capacitated sperm at a final concentration of $1 \times 10^{6} \mathrm{sperm} /$ $\mathrm{mL}$ (capacitation in the presence or absence of Cdc42 inhibitors). After coincubation for 4 hours at $37^{\circ} \mathrm{C}$ with $5 \% \mathrm{CO}_{2}$, eggs were washed three times and placed on drops containing TYH IVF at $37^{\circ} \mathrm{C}$ with $5 \% \mathrm{CO}_{2}$. Fertilization was assessed by the visualization of 2-cell embryos 20 hours later. 


\subsection{Statistical analysis}

Data are expressed as mean \pm standard error of the mean (SEM) of at least three independent experiments from different mice for all determinations. Statistical analyses were performed using the GraphPad Prism 6 software (La Jolla, CA USA). The differences between means of only two groups were analyzed using a t test (motility parameters, $\Delta \mathrm{Em}$ and 2-cell embryos). The non-parametric Kruskal-Wallis test was performed in combination with Dunn's multiple comparisons test to analyze normalized median fluorescence intensity (MFI) of Fluo-4 and normalized $I_{\text {CatSper }}$ currents. One-way analysis of variance (ANOVA) with Dunnett's multiple comparisons test was performed to analyze the percentage of sperm with high Fluo-4 fluorescence. A probability (p) value of $P<.05$ was considered statistically significant. Parametric or non-parametric comparisons were used as dictated by data distribution.

\section{3 | RESULTS}

\section{1 | Cdc42 localizes in four longitudinal lines along the flagellum resembling CatSper distribution pattern}

The presence of $\mathrm{Cdc} 42$ protein in mouse sperm was analyzed by immunoblotting using a specific antibody against this small GTPase. As shown in Figure 1A, A single band of the expected size for Cdc42 (21.3 kDa) was observed in these cells. Immunofluorescence studies revealed the localization of Cdc42 mainly along the flagellum as well as in the acrosome of mouse sperm (Figure 1B).

To investigate the spatial distribution of $\mathrm{Cdc} 42,3 \mathrm{D}$ STORM was used. This type of microscopy allows 3D reconstruction with $\sim 20 \mathrm{~nm}$ resolution, ${ }^{48,55}$ making it possible to study protein localization within the sperm flagellum, which is less than $1 \mu \mathrm{m}$ in diameter. ${ }^{42,47}$ The identification of specific structures within the flagellum was performed by analyzing the localization of well-known flagellar proteins. For the axoneme, the fibrous sheath and the plasma membrane, $\beta$-tubulin, A kinase anchoring protein 4 (AKAP4), and the facilitative glucose transporter 3 (GLUT3) were used as previously described ${ }^{42}$ (Supporting Information Figures S1 and S2). Taking advantage of the cylindrical symmetry of the flagellum, the molecule localizations were converted into cylindrical coordinates $\left(r, \Theta, \mathrm{z}^{\prime}\right)$, where $\mathrm{z}^{\prime}$ represents the flagellum axis. In cartesian coordinates $(\mathrm{x}, \mathrm{y}, \mathrm{z}), \mathrm{z}$ is the direction normal to the coverslip, so that in this coordinate system the sperm lies on the $\mathrm{x}, \mathrm{y}$ plane as represented in Supporting Information Figure S1A. As expected, the spatial distribution of $\beta$-tubulin was axonemal and localized in the center of the flagellum, while AKAP4 (localized in the fibrous sheath) and GLUT3 (present throughout the flagellar plasma membrane) showed a continuous rim distribution in cross-section (Supporting Information Figure S1B, right panel). The radial distributions of $\beta$-tubulin, AKAP4, and GLUT3 in the principal piece showed that $\beta$-tubulin peaked at $23 \pm 2 \mathrm{~nm}$ (mean \pm standard error) from the center of the flagellum, while AKAP4 and GLUT3 peaked at $146 \pm 1$ and $269 \pm 1 \mathrm{~nm}$, respectively (Supporting Information Figure S1C).

Mouse sperm exhibited a Cdc42 localization confined to four columns along the principal piece (Figure 1C and Supporting Information Video S1), which resembles the localization of CatSper in the same region. ${ }^{42}$ On crosssections of the flagellum, Cdc42 appeared as four tight puncta (Figure 1C right panel). Similar to Cdc42, P-CaMKII showed the CatSper-like distribution in the principal piece (Figure 1E,F and Supporting Information Figure S3C,D), as previously described. ${ }^{42}$ The radial distributions of Cdc42 and $\mathrm{P}-\mathrm{CaMKII}$ peaked at $238 \pm 16$ and $235 \pm 2 \mathrm{~nm}$, respectively (Figure 1D,F). These values are similar to those observed for GLUT3 (Supporting Information Figure S1B,C), indicating that both proteins localized close to the plasma membrane. A graphical representation of $\mathrm{Cdc} 42$ localization in terms of the azimuth angle $\theta$ vs axial distance $z$ ' along the principal piece showed the four stripes along the flagellum (Supporting Information Figure $\mathrm{S} 3 \mathrm{~A}$ ) as well as the 2D angular profiles of the surface localizations (Supporting Information Figure S3B). This segregated the localization of $\mathrm{Cdc} 42$ in the principal piece disappeared in the flagellum midpiece, where a continuous ring staining was observed in the cross-section (Figure 1G). The radial distribution in this section peaked at $281 \pm 25 \mathrm{~nm}$ (Figure 1H), while GLUT3 was observed at $323 \pm 2 \mathrm{~nm}$ in that region (Figure 1I,J).

Since the sperm flagellum is usually close to $1 \mu \mathrm{m}$ wide in the proximal portion and tapers gradually toward the distal tip, the molecular distribution of Cdc42 across the entire flagellum was visualized with 3D STORM. The localization of Cdc42 in four tight clusters was conserved along the principal piece (Figure 2). A series of cross-sections were analyzed (Figure 2B), where the decrease in $\mathrm{Cdc} 42$ radial distribution as the distance from the annulus increases was observed (Figure 2C).

Altogether, these results indicate that $\mathrm{Cdc} 42$ is organized in four columns in the principal piece and resembles the localization of CatSper and other signaling molecules reported in that structural domain.

\subsection{Cdc42 is delocalized in CatSper1 KO sperm}

It has been shown that the CatSper channel complex serves as an organizing structure for the four-line domains. In this respect, other molecules such as Caveolin-1, P-CaMKII, and PP2B lost their localization when CatSper is abrogated. ${ }^{42}$ Therefore, to test the extent to which $\mathrm{Cdc} 42$ is associated with 
(A)

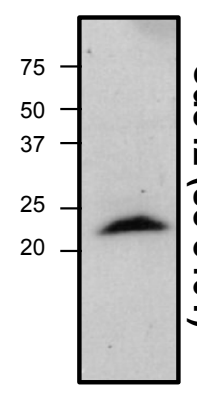

(B)

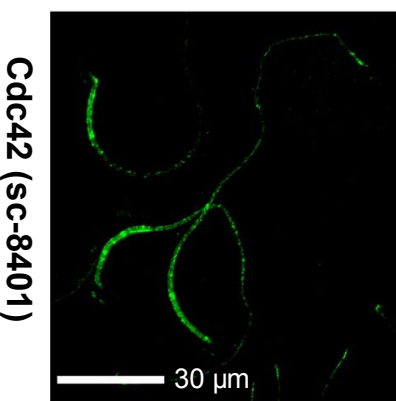

Cdc42

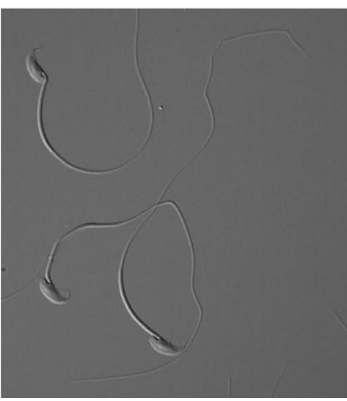

\section{Blocking Peptide}

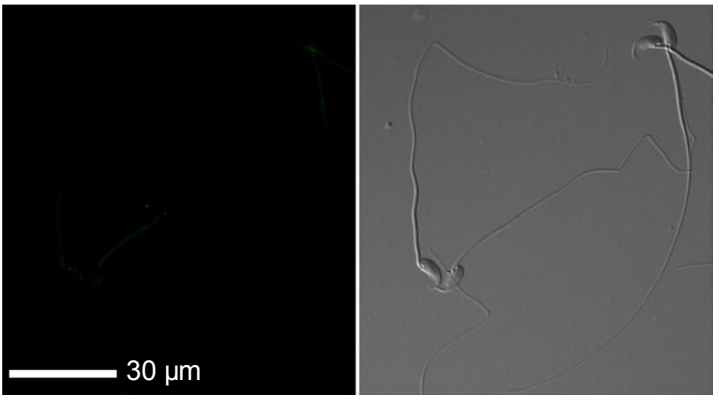

(C)

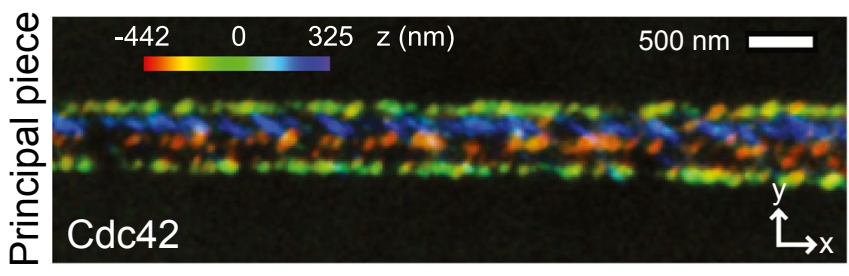

(E)

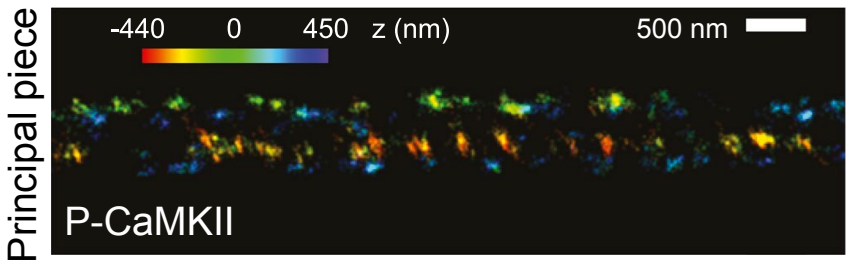

(G)

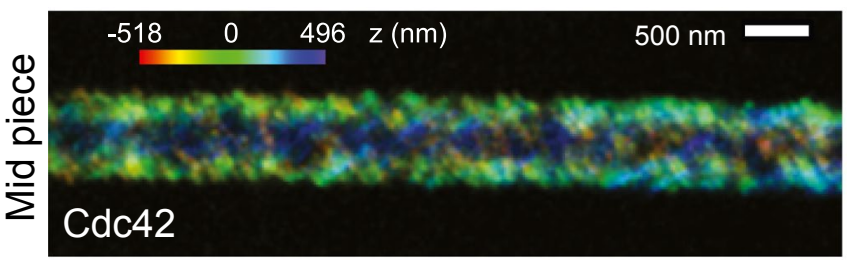

(I)

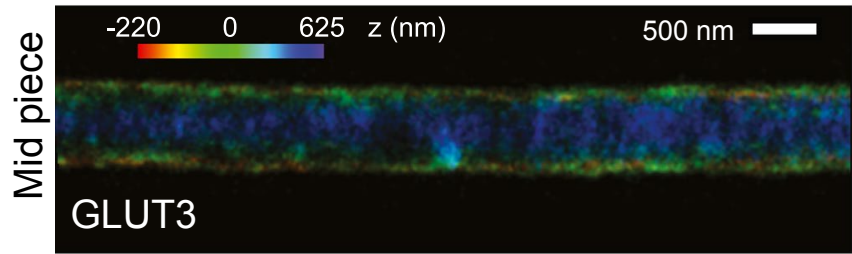

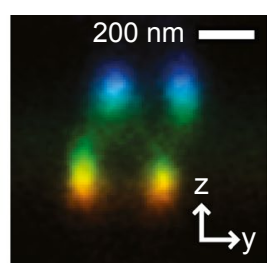

(D)

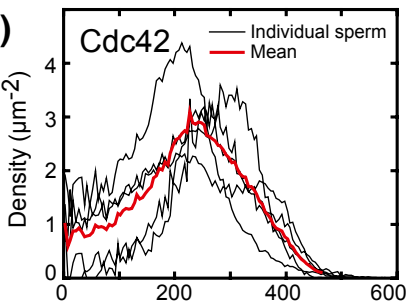

(F)
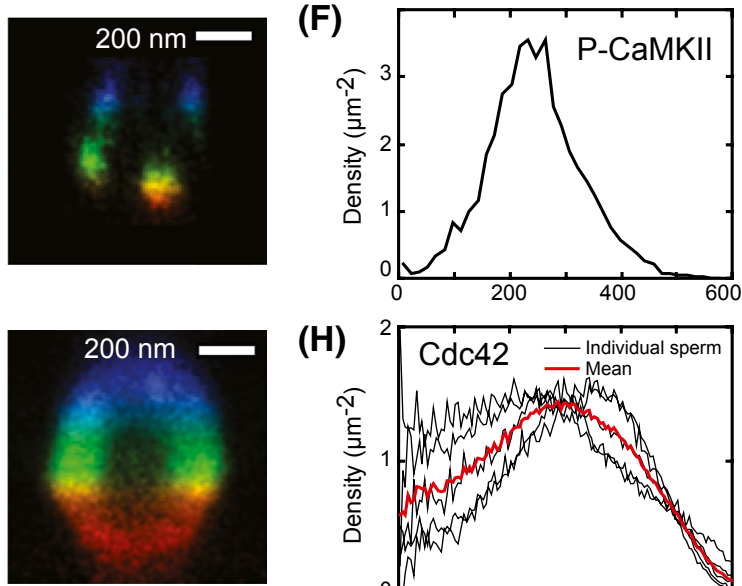

(H)

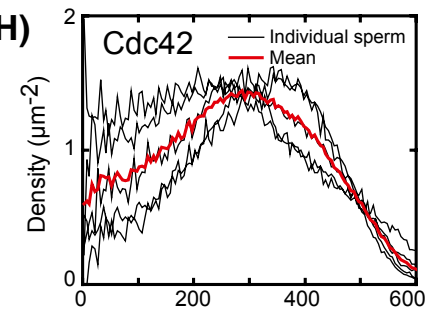

(J)

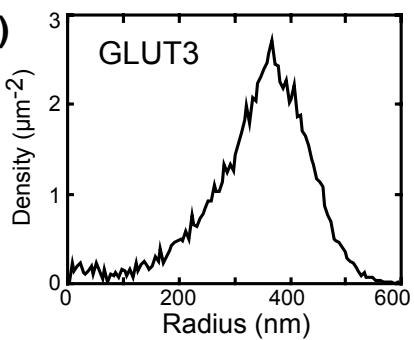

F I G URE 1 Cdc42 is localized in four longitudinal lines similar to those of the CatSper distribution. A, Proteins (equivalent to $14 \times 10^{6}$ sperm) were analyzed by $15 \%$ SDS-PAGE and immunoblotted using anti-Cdc42 (sc-8401). B, Representative immunofluorescence images and their corresponding phase-contrast micrographs of non-capacitated sperm stained with anti-Cdc42 antibody (sc-8401) are shown. Nonspecific staining was determined using blocking peptide (sc-8401 P). C, Representative 3D STORM images showing the localization of Cdc42 in the sperm principal piece at $\mathrm{x}-\mathrm{y}$ projection (left) and in $\mathrm{y}-\mathrm{z}$ cross-section (right). Anti-Cdc42 antibody (sc-8401) was used. D, Radial profiles of Cdc42 localization in the principal piece $(n=4$ sperm). E, Representative 3D STORM images of principal piece P-CaMKII. Left, $\mathrm{x}-\mathrm{y}$ projections. Right, $y$-z cross-sections. F, Radial profiles of P-CaMKII localization in the principal piece. G, Representative 3D STORM images showing the localization of $\mathrm{Cdc} 42$ in the sperm midpiece at $\mathrm{x}-\mathrm{y}$ projection (left) and in $\mathrm{y}-\mathrm{z}$ cross-section (right). H, Radial profiles of the immunostained density of the sperm midpiece localized Cdc42. I, Representative 3D STORM images of midpiece GLUT3. Left, $x-y$ projections. Right, $y-z$ cross-sections. $\mathrm{J}$, Radial profiles of the immunostained density of GLUT3. The color in all x-y projections encodes the relative distance from the focal plane along the $\mathrm{z}$ axis 
(A)

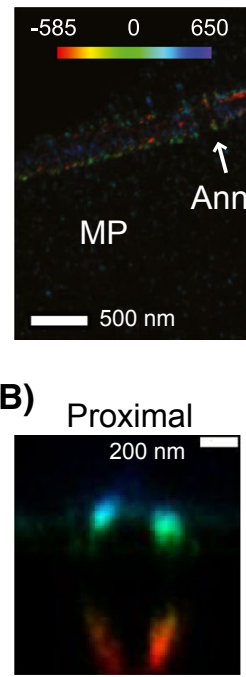

0.05

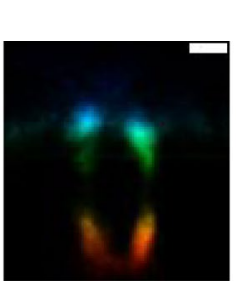

3.4

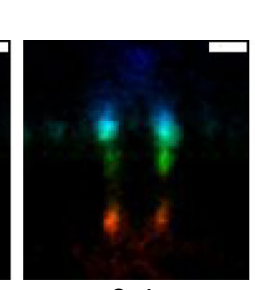

9.4
(C)

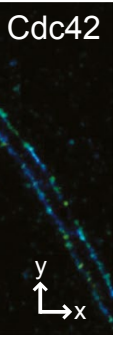

Distal

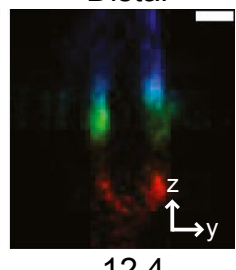

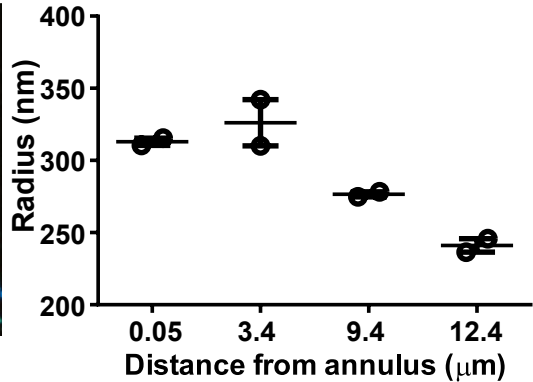

Distance from annulus $(\mu \mathrm{m})$

F I G URE 2 Cdc42 is localized in four longitudinal lines along the principal piece. A, Representative 3D STORM images showing the localization of Cdc42 along the sperm principal piece. Anti-Cdc42 antibody (sc-8401) was used. Entire x-y field of view. MP, midpiece. PP, principal piece. An arrow marks the annulus. The color encodes the relative distance from the focal plane along the $\mathrm{z}$ axis. B, Series of magnified $\mathrm{y}-\mathrm{z}$ cross-sections obtained from the proximal to the distal part of the principal piece (same cell). Distance from annulus ( $\mu \mathrm{m}$ ) is specified. C, The radius $(\mathrm{nm})$ of $\mathrm{Cdc} 42$ staining was calculated in the principal piece at different distances from the annulus. Values represent the mean $\pm \mathrm{SEM}$ of two measurements

this complex, its localization in sperm from CatSper1-null mice was analyzed. Levels of protein expression in CatSper1 KO sperm were examined by immunoblotting using two specific antibodies (Figure 3A,B). Expression of Cdc42 was detected in CatSper1 KO, albeit at 50\% lower levels than in HET sperm (Figure 3A,B, right panel). Immunofluorescence studies revealed that $\mathrm{Cdc} 42$ was also present along the flagellum and in the acrosome of CatSper1 KO (Figure 3C) as in the HET sperm; although with lower fluorescence intensity, consistent with reduced protein expression (Figure 3A,B). 3D STORM analyses in a principal piece from CatSper1 KO sperm were performed, but only poor-quality reconstructions could be obtained due to the low number of localized fluorophores $(2 \pm 1$ localizations/ $\mathrm{nm}$ in CatSper1 KO vs $10 \pm 2$ localizations/nm in HET sperm), in agreement with lower protein expression. In summary, 21 out of 23 sperm analyzed of CatSper1 KO sperm showed a clear Cdc42 delocalization from the quadrilateral structure in comparison with HET sperm (Figure 3D). Altogether, these results suggest that the absence of CatSper channels alters the expression levels and the spatial 3D distribution of $\mathrm{Cdc} 42$.

\section{3 | Inhibition of $\mathrm{Cdc42}$ decreases $\left[\mathrm{Ca}^{2+}\right]_{\mathrm{i}}$ during capacitation}

Given the Cdc42 distribution in four linear domains in the principal piece (Figure 1C,D), we postulated that this signaling protein could be involved in the CatSper function. This hypothesis was tested by different approaches using a pharmacological inhibitor. Cdc42 activity is highly regulated by switching between its active GTP-bound form and its inactive GDP-bound form. This modulation depends on the exchange of GDP to GTP by guanine nucleotide exchange factors (GEF), whereas GTPase activating proteins (GAP) bind to active $\mathrm{Cdc} 42$ stimulating the GTP-hydrolytic reaction, thus terminating the signaling event. ${ }^{56}$ In addition, guanine nucleotide dissociation inhibitors (GDI) prevent GEF-mediated nucleotide exchange, maintaining the GTPase in an inactive state and promoting $\mathrm{Cdc} 42$ dissociation from the membrane. ${ }^{57}$ To assess the function of Cdc42 in mouse sperm, MLS-573151, a specific pharmacological inhibitor that prevents the binding of GTP to $\mathrm{Cdc} 42^{58}$ was used.

To analyze the participation of Cdc 42 on CatSper activity, $\left[\mathrm{Ca}^{2+}\right]_{\mathrm{i}}$ was evaluated by flow cytometry in the presence or absence of MLS-573151. We recently determined by flow cytometry that the capacitation-associated $\left[\mathrm{Ca}^{2+}\right]_{\mathrm{i}}$ increase at 90 minutes was dependent on CatSper channels, as sperm derived from CatSper1 KO failed to increase $\left[\mathrm{Ca}^{2+}\right]_{\mathrm{i}} \cdot{ }^{17}$ Thus, $\left[\mathrm{Ca}^{2+}\right]_{\mathrm{i}}$ was measured by flow cytometry in live sperm loaded with the $\mathrm{Ca}^{2+}$ sensitive probe Fluo-4 AM. Sperm were incubated for 90 minutes under capacitating conditions in the presence of increasing concentrations of MLS-573151. The addition of MLS-573151 significantly suppressed the rise in 
(A)

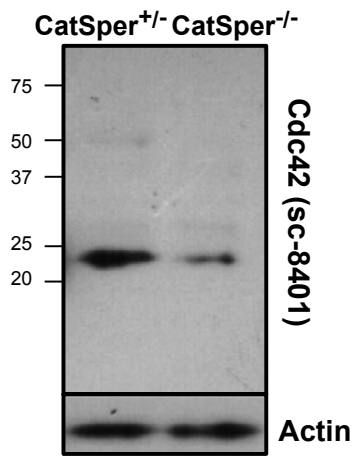

(B)

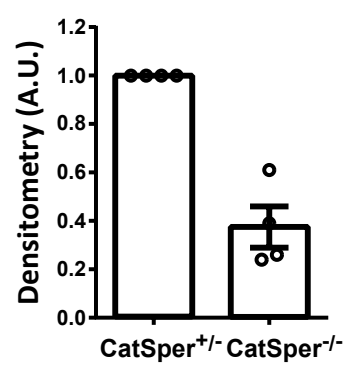

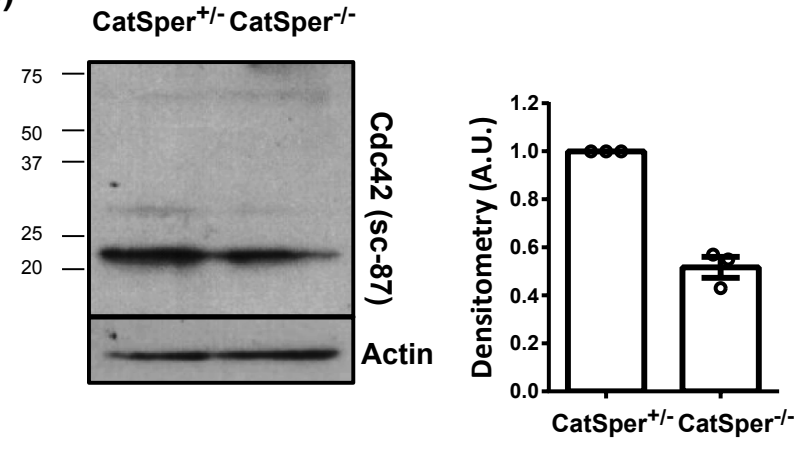

(C)

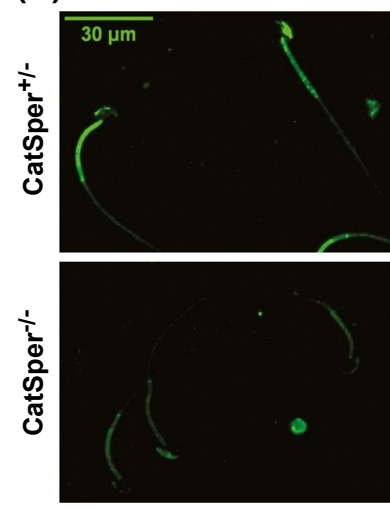

(D)

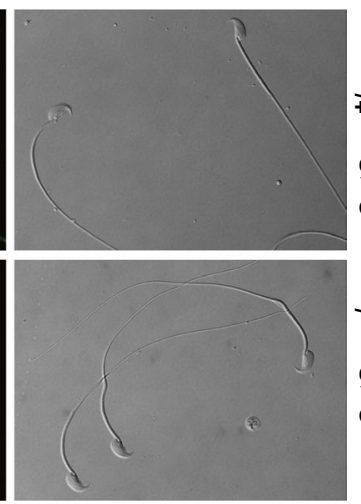

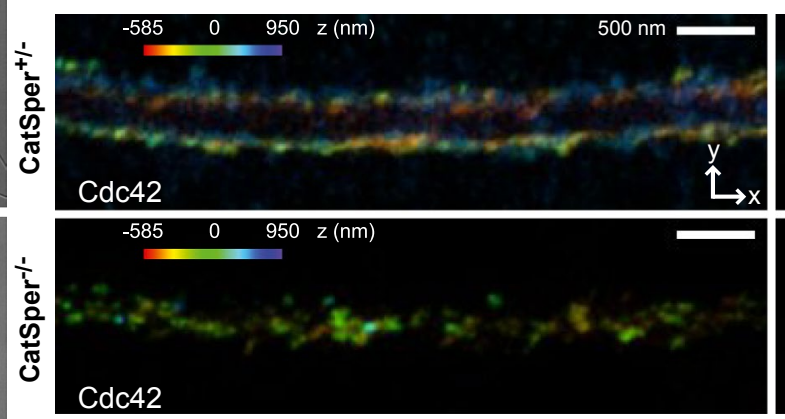

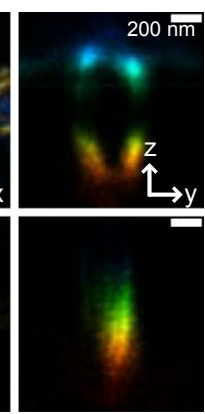

F I G U RE 3 CatSper is essential for Cdc42 expression and spatial organization. A, Proteins (equivalent to $12 \times 10^{6}$ sperm) were analyzed by $15 \%$ SDS-PAGE and immunoblotted using anti-Cdc42 (sc-8401). As a loading control, an anti-Actin antibody was used. Values represent the mean \pm SEM of four independent experiments, where normalization to the control condition (CatSper ${ }^{+/-}$sperm) was used. Non-parametric Wilcoxon signed-rank test was performed. B, Proteins (equivalent to $10 \times 10^{6}$ sperm) were analyzed by $15 \%$ SDS-PAGE and immunoblotted using anti-Cdc42 (sc-87). As a loading control, an anti-Actin antibody was used. Values represent the mean \pm SEM of three independent experiments, where normalization to the control condition (CatSper $1^{+/-}$sperm) was used. Non-parametric Wilcoxon signed-rank test was performed. C, Representative immunofluorescence images and the corresponding phase-contrast micrographs of non-capacitated control $\left(\mathrm{CatSper1}^{+/-}\right)$and CatSper1 KO (CatSper1 ${ }^{-/}$) sperm stained with anti-Cdc42 antibody (sc-8401) are shown. D, Representative 3D STORM images showing the localization of $\mathrm{Cdc} 42$ in the principal piece of control $\left(\right.$ CatSper $1^{+/-}$) sperm at x-y projection (left, upper panel) and in y-z cross-section (right, upper panel). Representative 3D STORM images of CatSper1 KO (CatSper1 ${ }^{-/-}$) principal piece Cdc42 (lower panel). Anti-Cdc42 antibody (sc-8401) was used. Left, $x$-y projections. Right, $y$-z cross-sections $(n=23$ sperm from three different mice). The color in all $x-y$ projections encodes the relative distance from the focal plane along the $\mathrm{z}$ axis

$\left[\mathrm{Ca}^{2+}\right]_{\mathrm{i}}$ that occurs during capacitation as a result of CatSper activation (Figure 4A,B) in a concentration-dependent manner (Figure 4C and Supporting Information Figure S4). The concentration of $20 \mu \mathrm{M}$ of MLS-573151 was chosen for all the experiments as the $\left[\mathrm{Ca}^{2+}\right]_{\mathrm{i}}$ blockage was maximum without changing sperm viability. This inhibition can be visualized by a decrease in the normalized median fluorescence intensity (MFI) of Fluo-4 (Supporting Information Figure S4) as well as in the percentage of sperm that displayed a rise in $\left[\mathrm{Ca}^{2+}\right]_{\mathrm{i}}$ (Figure 4C). The population of sperm with high fluorescence was determined from the capacitating control condition (CAP 90 minutes) and extrapolated to the other conditions of each experiment (Figure 4B).

To corroborate that MLS-573151 is inhibiting Cdc42 activity, an ELISA-based assay that measure the GTPbound form of $\mathrm{Cdc} 42$ was performed. As expected, sperm capacitated in the presence of MLS-573151 significantly decreased the Cdc42 active form (Figure 4D).

\subsection{Cdc42 activity is required for hyperactivation and in vitro fertilization}

$\mathrm{Ca}^{2+}$ brought in from CatSper channels is essential for hyperactivation and successful fertilization. ${ }^{21}$ Given the decrease in $\left[\mathrm{Ca}^{2+}\right]_{\mathrm{i}}$ observed as a result of Cdc42 inhibition, hyperactivated motility and in vitro fertilization were assessed in the presence of MLS-57151. For this purpose, motility patterns were analyzed by computer-assisted semen analysis (CASA) in those samples exposed to Cdc 42 inhibitor or control capacitating medium. Cdc42 inhibition by MLS-573151 produced a significant decrease in 
the percentage of hyperactivated cells when compared with controls (Figure 4E). This decrease was also observed in the kinetic parameters evaluated, in particular, in those that are used to identify the hyperactivated population such as VCL, LIN, and ALH (Supporting Information Table S1). Although a decrease in sperm motility was observed with MLS-573151 (Supporting Information Table S1), the high percentage of motile sperm observed after exposure to
Cdc42 inhibitor (more than $80 \%$ ) indicated that sperm viability was not affected by this compound.

Next, in vitro fertilization of cumulus-oocyte complexes (COC) was performed using sperm that were capacitated in the presence or absence of $\mathrm{Cdc} 42$ inhibitor, and the percentage of fertilized eggs was determined. Fertilization rates were significantly lower when Cdc42 was inhibited by MLS573151, supporting its role in capacitation (Figure 4F).
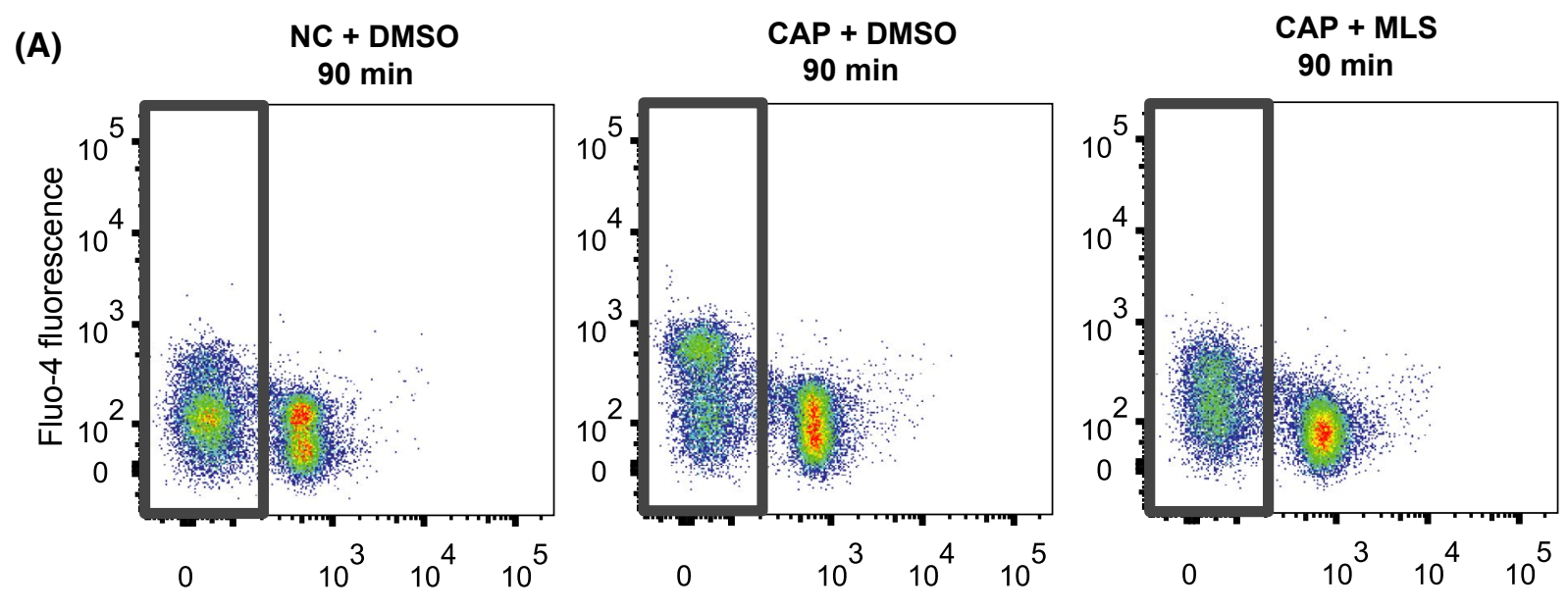

(B)

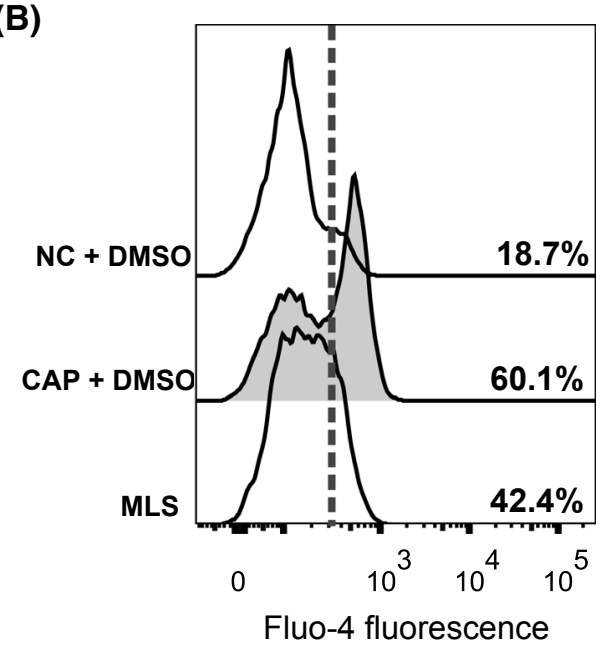

(D)

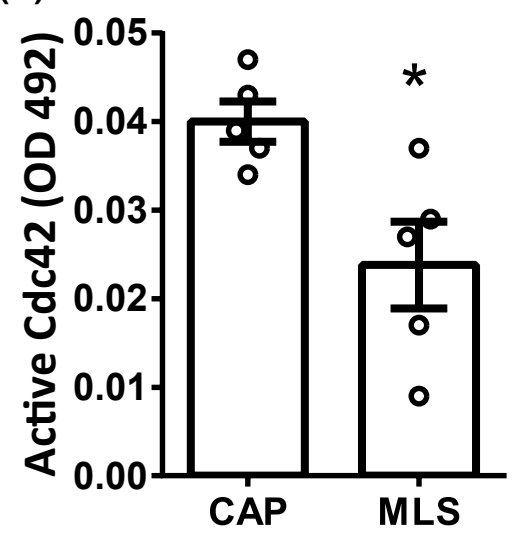

(E)

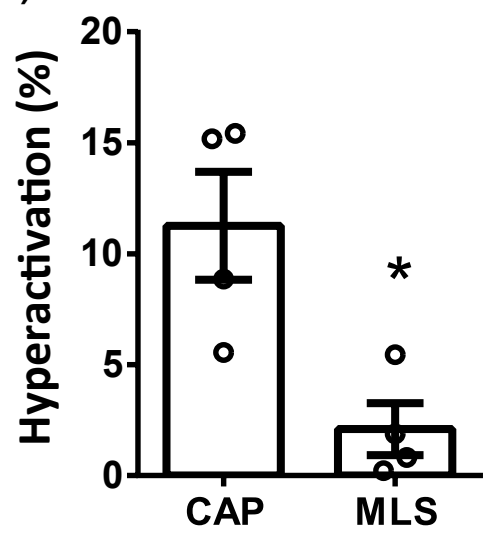

(C)

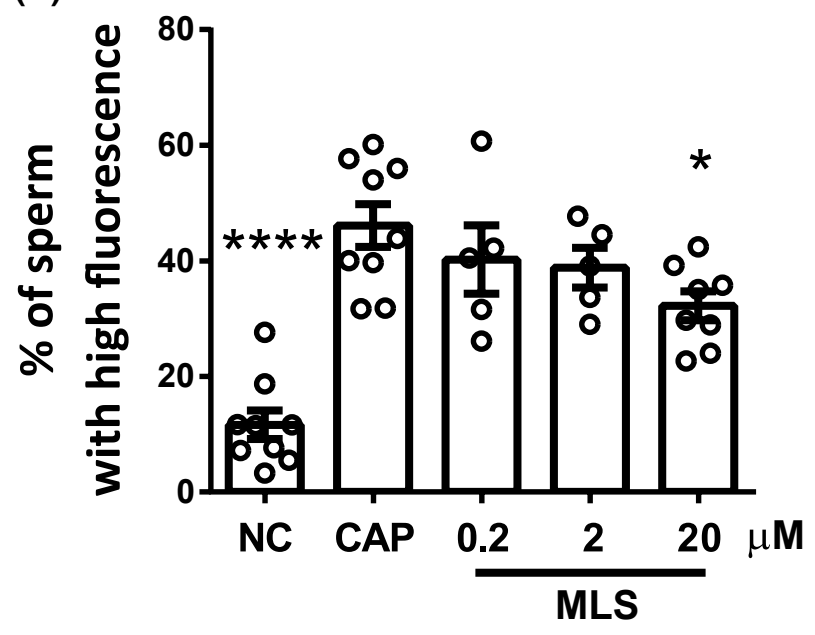

(F)

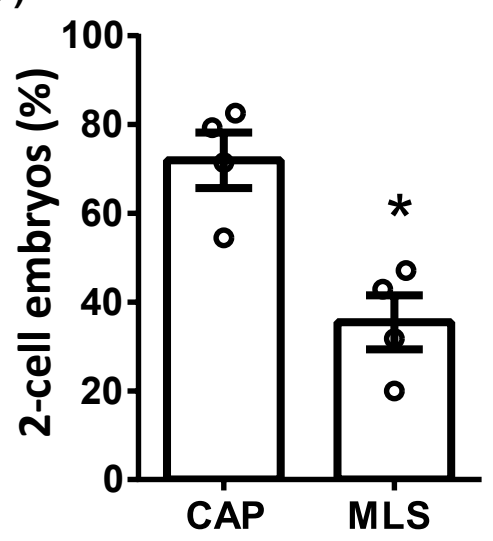


F I G URE 4 Capacitation-associated $\left[\mathrm{Ca}^{2+}\right]_{\mathrm{i}}$ increase, hyperactivation and in vitro fertilization were blocked by Cdc42 inhibitor. Sperm incubated for 90 minutes under non-capacitating (NC) or capacitating conditions (CAP) in the absence (DMSO) or presence of $20 \mu \mathrm{M}$ of MLS573151 were analyzed. A, Representative two-dimensional Fluo-4 vs PI fluorescence dot plots are shown. Live sperm were identified (delimited by a gray rectangle) from which Fluo-4 fluorescence levels were analyzed in order to establish $\left[\mathrm{Ca}^{2+}\right]_{\mathrm{i}}$. B, Representative histograms of normalized frequency vs Fluo-4 fluorescence of non-PI stained sperm (live), with the corresponding percentage of sperm that increased Fluo-4 fluorescence, are shown. The percentage of sperm that responds by increasing the $\left[\mathrm{Ca}^{2+}\right]_{\mathrm{i}}$ was established in the CAP control condition and extrapolated to the others conditions (dashed line). C, Percentage of sperm that increased Fluo-4 fluorescence after being incubated for 90 minutes in the different conditions. Values represent the mean \pm SEM of nine independent experiments. $* * * * P<.0001 ; * P<.05$ represents statistical significance. One-way ANOVA with Dunnett's multiple comparisons test was performed against the control condition: CAP 90 minutes with DMSO. D, Quantification of active (GTP-bound) Cdc42 in the absence (DMSO) or presence of $20 \mu \mathrm{M}$ of MLS-573151. E, Percentage of hyperactive sperm (\%) in the absence (DMSO) or presence of $20 \mu \mathrm{M}$ of MLS-573151. F, Fertilization rates after in vitro fertilization (percentage of 2-cell embryos per total oocytes examined) in the absence (DMSO) or presence of $20 \mu \mathrm{M}$ of MLS-573151. Data represent the mean \pm SEM of at least four independent experiments. $* P<.05$ represents statistical significance vs control (CAP 90 minutes with DMSO). Paired t test was performed

\subsection{Cdc42 activity is essential for CatSper function}

To determine whether Cdc 42 activity is required for CatSper function, we used a fluorescence method as previously described. ${ }^{54}$ This assay is based on the fact that removing external $\mathrm{Ca}^{2+}$ and $\mathrm{Mg}^{2+}$ after adding EGTA allows CatSper to efficiently conduct monovalent cations ${ }^{39}$ where a sudden influx of $\mathrm{Na}^{+}$depolarizes the cells. ${ }^{59,60}$ The magnitude of this depolarization mainly depends on the extent of CatSper opening and is inhibited by CatSper channel blockers. ${ }^{54,60}$ Sperm membrane potential (Em) was measured with a fluorescent cyanine dye $\mathrm{DiSC}_{3}(5)$. Because this cationic dye accumulates on cells with hyperpolarized membranes, an increase in $\operatorname{DiSC}_{3}(5)$ fluorescence in the medium reflects Em depolarization. Thus, after EGTA addition, the magnitude of the Em depolarization was visualized as changes in $\operatorname{DiSC}_{3}(5)$ fluorescence in the extracellular medium. Incubation with $\mathrm{Cdc} 42$ inhibitor during capacitation significantly diminished the Em depolarization caused by EGTA addition (Figure 5A,B), suggesting that CatSper opening is affected by inhibiting Cdc42.

CatSper channels are strongly activated by an intracellular alkalinization. ${ }^{39,40}$ To evaluate if $\mathrm{Cdc} 42$ alters the sperm intracellular $\mathrm{pH}\left(\mathrm{pH}_{\mathrm{i}}\right)$, this parameter was analyzed in live cells by flow cytometry, loading sperm with the $\mathrm{pH}_{\mathrm{i}}$ sensitive probe BCECF AM. Cells were incubated for 90 minutes under non-capacitating and capacitating conditions in the presence or absence of Cdc42 inhibitor. The intracellular alkalinization associated with capacitation was not modified by the presence of MLS-573151 (Figure 5C).

To better characterize the Cdc42-dependent CatSper gating, whole-cell voltage-clamp recordings of CatSper currents $\left(I_{\text {Catsper }}\right)$ were performed. $I_{\text {CatSper }}$ from cauda epididymal sperm were analyzed using a typical voltage-ramp protocol (from -80 to $+80 \mathrm{mV}$ during $750 \mathrm{~ms}$ with a holding potential of $0 \mathrm{mV}$ ) and $\mathrm{Na}^{+}$out/ $/ \mathrm{Cs}^{+}$in as the main conducting ions in the absence of divalent cations (divalent cation-free condition, DVF). A concentration of $0.4 \mathrm{mM}$ GTP was added to the recording pipette solution to ensure that the activity of Cdc42 is not limited by the lack of this cofactor. Under non-capacitating conditions, the $I_{\text {Catsper }}$ was not altered by the presence of MLS-573151 (Figure 5D,E). However, while $I_{\text {CatSper }}$ was strongly stimulated under capacitating conditions, this current was potently decreased by the addition of MLS573151 (Figure 5F,G). Altogether, these results suggest that Cdc42 activity is necessary for CatSper function, by a mechanism different from $\mathrm{pH}_{\mathrm{i}}$ regulation.

There is evidence that CatSper channels are promiscuous and may be inhibited by different compounds in a non-specific manner. ${ }^{61,62}$ To validate our results, we explored the specificity of the Cdc42 inhibitor used in previous experiments. To this aim, we used a condition where $\mathrm{Cdc} 42$ activity is compromised (absence of GTP) and took advantage of the fact that mouse CatSper is strongly activated by intracellular alkalinization (promoted by $\mathrm{NH}_{4} \mathrm{Cl}$ addition), as demonstrated by an increase in $I_{\text {CatSper }}{ }^{39}$ The mechanism for $\mathrm{pH}$-dependent activation is not yet fully understood but it has been reported that the protein EFCAB9 together with the highly rich histidine CatSper amino-terminus domain is responsible for sensing changes in $\mathrm{pH}_{\mathrm{i}} \cdot{ }^{21,30}$ Our experiment is based on the premise that if CatSper is non-specifically blocked by the action of Cdc42 inhibitor, alkalinization would not produce a robust increase in CatSper activity.

In order to analyze whether $\mathrm{Cdc} 42$ modulation on CatSper disturbs $\mathrm{pH}_{\mathrm{i}}$ activation, non-capacitated sperm loaded with the Em sensitive probe $\operatorname{DiSC}_{3}(5)$ were first exposed to $\mathrm{Cdc} 42$ inhibitor and then to $\mathrm{NH}_{4} \mathrm{Cl}$. CatSper opening was evidenced by the magnitude of Em depolarization resulting from $\mathrm{Ca}^{2+}$ influx. MLS-573151 pre-incubation did not alter CatSper sensitivity to $\mathrm{pH}$ alkalinization (Figure 6A,B).

To further corroborate this result, $I_{\text {CatSper }}$ was analyzed under non-capacitating conditions, without the addition of GTP in the recording patch-clamp pipette solution, where the $I_{\text {Catsper }}$ was not altered by MLS-573151 (Figure 6C,D). The increase in $I_{\text {Catsper }}$ as a result of CatSper activation induced by $\mathrm{pH}_{\mathrm{i}}$ alkalinization was obtained by adding $\mathrm{NH}_{4} \mathrm{Cl}(10 \mathrm{mM})$, and this stimulation was not modified by the presence of 
(A)

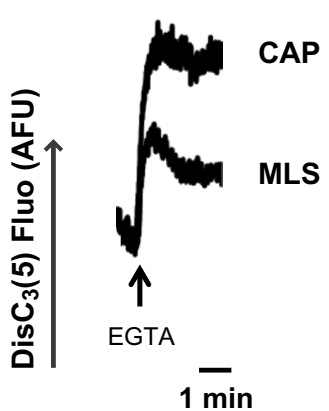

(B)

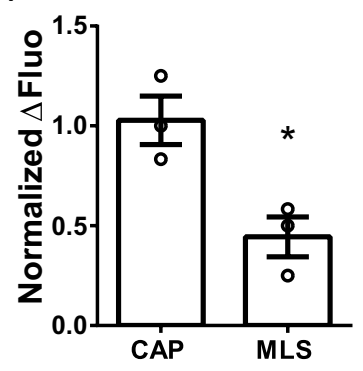

(C)

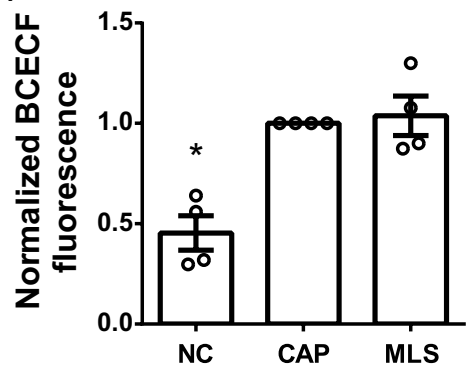

(D)

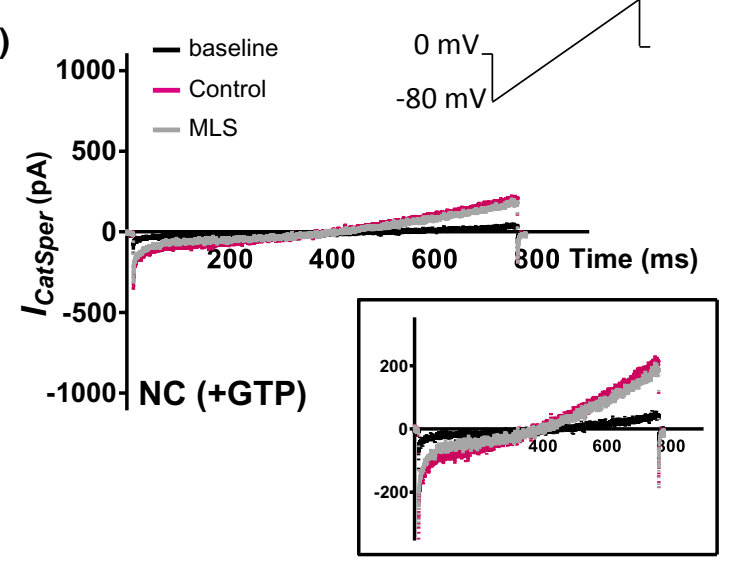

(E)

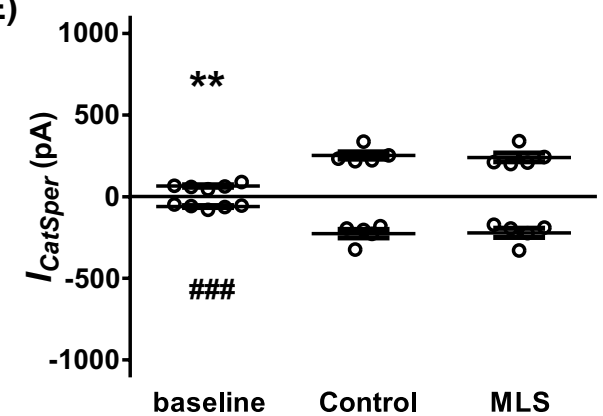

(F)

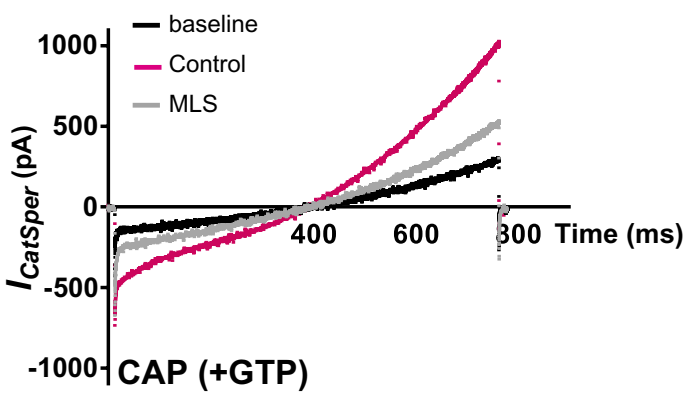

(G)

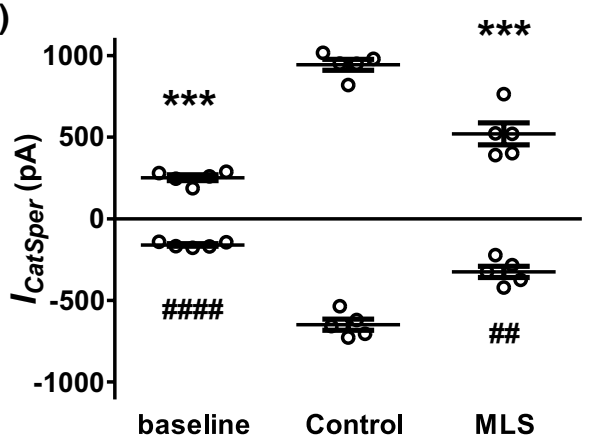

F I G URE 5 Capacitation-associated increase in CatSper currents was block by Cdc42 inhibitor. A, Sperm were incubated for 90 minutes under capacitating conditions (CAP) in the absence (DMSO) or presence of $10 \mu \mathrm{M}$ MLS-573151. CatSper activity was analyzed by measuring Em with $\mathrm{DiSC}_{3}(5)$, before and after the addition of $3.5 \mathrm{mM}$ EGTA. Representative DiSC $\mathrm{C}_{3}(5)$ fluorescence traces (AFU: arbitrary fluorescence units) through time are shown. $\mathrm{B}$, Normalized $\Delta$ Fluorescence represents the difference between $\mathrm{DiSC}_{3}(5)$ fluorescence after EGTA addition $\left(\mathrm{F}_{\mathrm{EGTA}}\right)$ and before (resting: $\mathrm{F}_{\mathrm{R}}$ ) compared to the mean obtained in the control condition (CAP 90 minutes with DMSO). Data represent the mean \pm SEM of three independent experiments. ${ }^{*} P<.05$ represents statistical significance vs control (CAP 90 minutes with DMSO). Unpaired t test was performed. C, Mouse sperm $\mathrm{pH}_{\mathrm{i}}$ was measured by BCECF AM and flow cytometry. Sperm incubated for 90 minutes under NC or CAP conditions in the absence (DMSO) or presence of $20 \mu \mathrm{M}$ MLS-573151 were analyzed. Normalized MFI of BCECF compared to the control condition (CAP 90 minutes with DMSO). Values represent the mean \pm SEM of four independent experiments. $* P<.05$ represents statistical significance vs control (CAP 90 minutes with DMSO). Non-parametric Kruskal-Wallis test was performed in combination with Dunn's multiple comparisons test. D and F, Representative whole-cell currents traces from non-capacitated (D) and capacitated (F) mouse sperm. Inward and outward currents were elicited by a voltage ramp protocol from a holding potential of $0 \mathrm{mV}$ (inset). To ensure stable recording conditions, first baseline currents (in HS solution) were obtained. Under HS condition (black traces) CatSper currents were minimal. In cation divalent-free (DVF) medium (magenta traces) typical CatSper monovalent currents can be recorded. In the presence of $20 \mu \mathrm{M}$ MLS-573151 (light gray traces), CatSper currents were inhibited only under CAP conditions. The patch pipette was always filled with $0.4 \mathrm{mM} \mathrm{GTP}$. E and G, Quantification of $I_{\text {CatSper }}$ current densities for all three conditions. Data represent the mean \pm SEM of five sperm from different mice. $* * * P<.001 ; * * P<.01$ represents statistical significance vs DVF control $(+80 \mathrm{mV}) .{ }^{\# \# \#} P<.0001 ;{ }^{\# \# \#} P<.001 ;{ }^{\# \#} P<.01$ represents statistical significance vs DVF control $(-80 \mathrm{mV})$. One-way ANOVA was performed in combination with Holm-Sidak's multiple comparisons test 
(A)

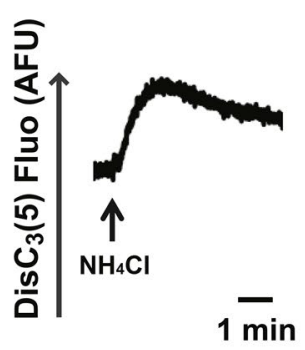

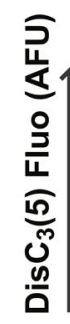

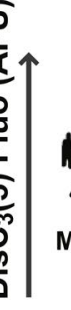

(B)

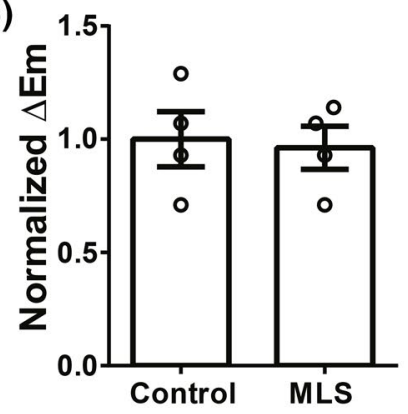

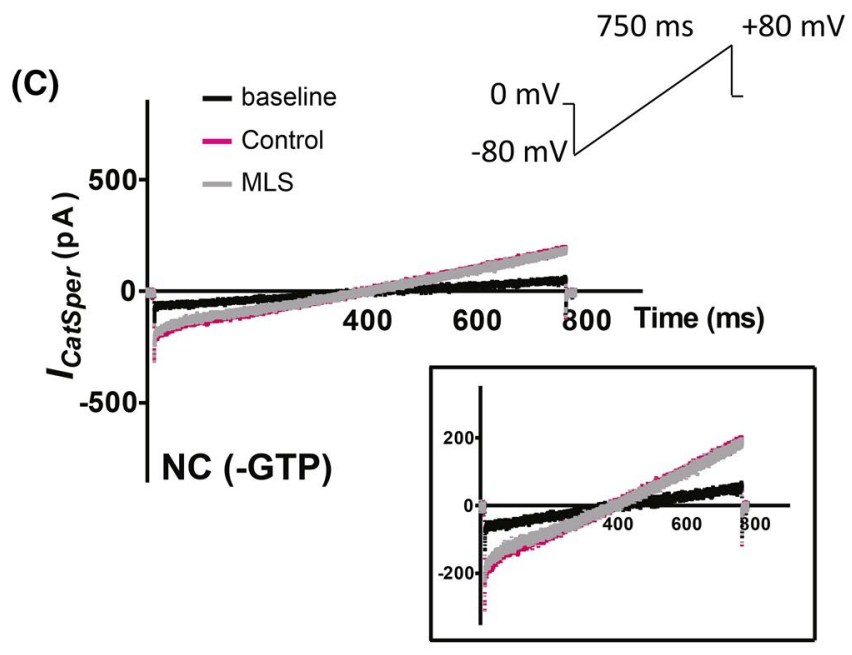

(D)
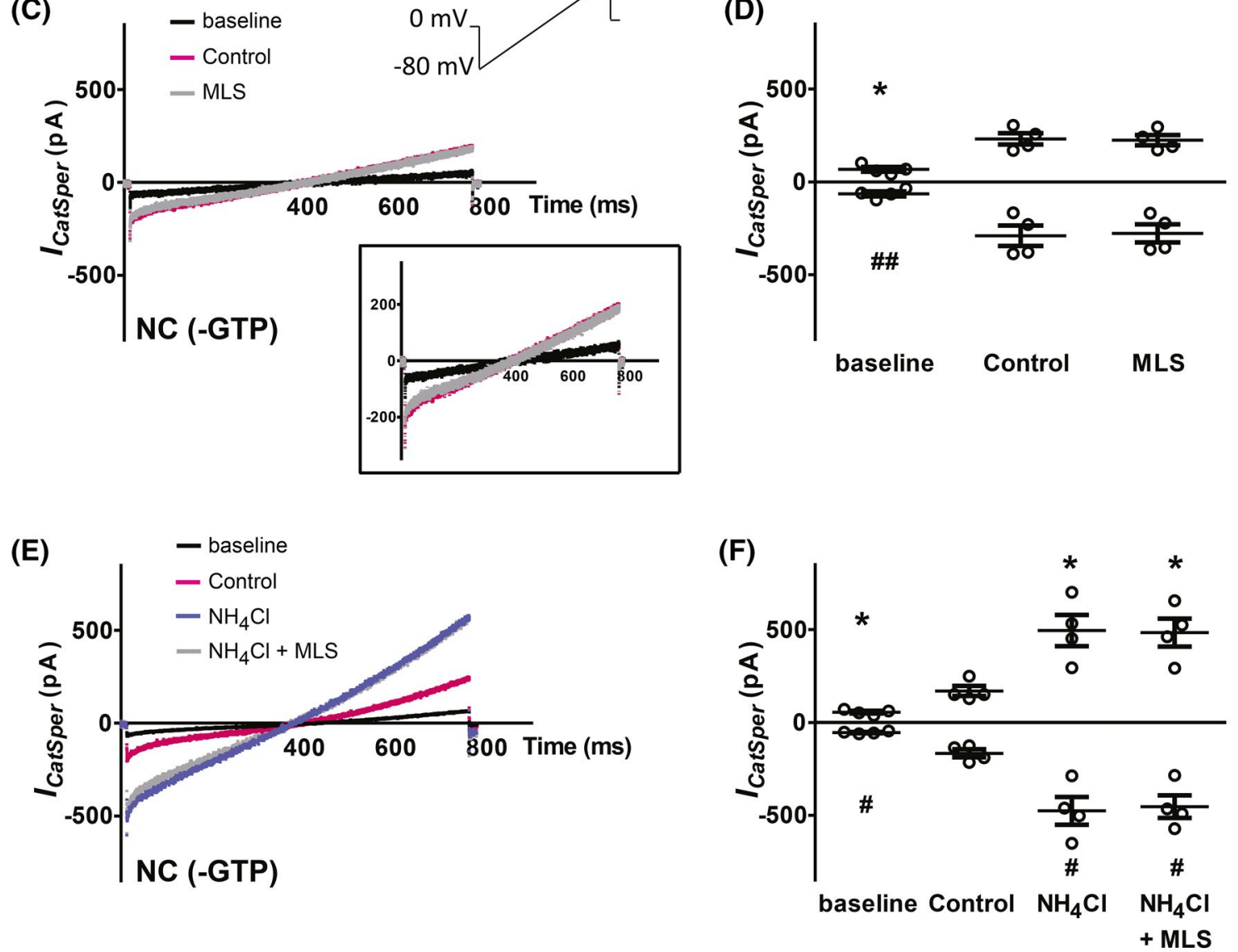

F IG U RE 6 Alkalinization-activated CatSper current is not modified by MLS-573151. CatSper activity was analyzed by measuring Em with $\operatorname{DiSC}_{3}(5)$ in a population assay. A, Representative fluorescence traces (AFU: arbitrary fluorescence units) of Em through time are shown. The addition of $10 \mathrm{mM} \mathrm{NH}_{4} \mathrm{Cl}$ activates the CatSper channel causing depolarization by $\mathrm{Ca}^{2+}$ influx (left traces). Addition of $10 \mu \mathrm{M}$ MLS-573151 previous to $\mathrm{NH}_{4} \mathrm{Cl} 10 \mathrm{mM}$ did not modified CatSper activation (right traces). B, Normalized $\Delta \mathrm{Em}$ represents the difference between Em after $\mathrm{NH}_{4} \mathrm{Cl}$ addition $\left(\mathrm{Em}_{\mathrm{NH} 4 \mathrm{Cl}}\right)$ and before (resting: $\mathrm{Em}_{\mathrm{R}}$ or $\mathrm{Em}_{\mathrm{Cdc} 42 \text { inhibitor }}$ ) compared to the mean obtained in the control condition (without $\mathrm{Cdc} 42$ inhibitor addition). Data represent the mean \pm SEM of at least three independent experiments. $\Delta E \mathrm{~m}$ of $\mathrm{NC}=14.91 \pm 0.96 \mathrm{mV}(\mathrm{n}=11)$. Unpaired $\mathrm{t}$ test was performed. $\mathrm{C}$ and $\mathrm{E}$, Representative whole-cell currents traces from non-capacitated mouse sperm without the addition of GTP to the recording pipette. Inward and outward currents were elicited by a voltage ramp protocol from a holding potential of $0 \mathrm{mV}$ (inset). To ensure stable recording conditions, first baseline currents (in HS solution) were obtained. Under HS condition (black traces) CatSper currents were minimal. In cation divalent-free (DVF) medium (magenta traces) typical CatSper monovalent currents can be recorded. C, In the presence of $20 \mu \mathrm{M}$ MLS573151 (light gray traces), CatSper currents were not modified. E, DVF after adding $10 \mathrm{mM} \mathrm{NH}_{4} \mathrm{Cl}$, with (light gray traces) or without (light blue traces) $20 \mu \mathrm{M}$ MLS-573151. D and F, Quantification of $I_{\text {CatSper }}$ current densities. Data represent the mean \pm SEM of four sperm from different mice. ${ }^{*} P<.05$ represents statistical significance vs DVF control $(+80 \mathrm{mV}) .{ }^{\# \#} P<.01 ;{ }^{\#} P<.05$ represents statistical significance vs DVF control $(-80 \mathrm{mV})$. One-way ANOVA was performed in combination with Holm-Sidak's multiple comparisons test 
MLS-573151 (Figure 6E,F). Overall, MLS-573151 did not directly affect $I_{\text {CatSper }}$ nor the alkalinization-induced Em depolarization under these experimental conditions. These results support that this inhibitor is not producing a nonspecific blockade of CatSper channels and may be safely used to investigate the role of $\mathrm{Cdc} 42$ in mouse sperm.

In addition to MLS-573151, other two specific pharmacological inhibitors were also used to study Cdc42. Secramine A blocks the membrane recruitment of $\mathrm{Cdc} 42$ by impeding the release of GDI from Cdc42 (Cdc42/GDI complex), which sequesters the inactive GDP-bound Cdc42 in the cytosol and inhibits Cdc42 activation (GTP loading). ${ }^{63}$ CASIN targets Cdc42 by specifically blocking GEF activity on the Cdc42/GDI complex (referred to in ${ }^{64}$ as pirl1-related compound 2). On one hand, Secramine A partially impairs alkalinization-dependent CatSper activation, observed by a decrease in Em depolarization after $\mathrm{NH}_{4} \mathrm{Cl}$ addition in comparison with the control condition (without Cdc42 inhibitor) (Supporting Information Figure S5A,B). Moreover, the addition of Secramine A completely inhibited the alkalinization stimulated $I_{\text {Catsper }}$ (Supporting Information Figure S5C,D). On the other hand, because CASIN provoked a significant Em depolarization itself, it was not possible to observe the change in Em produced by $\mathrm{NH}_{4} \mathrm{Cl}$ (Supporting Information Figure S5E,F); however, CASIN decreased the $I_{\text {Catsper }}$ partially ( $50 \%$ ) (Supporting Information Figure S5G,H) at negative and positive voltages. These findings show that both $\mathrm{Cdc} 42$ inhibitors have off-target effects on CatSper activity and are not suitable to evaluate the role of $\mathrm{Cdc} 42$ on mouse sperm physiology.

\subsection{Cdc42 is necessary for cAMP production by $\mathrm{SAC}$}

Our results identify Cdc 42 as a new endogenous regulator of CatSper. Recently, it was demonstrated that CatSper activity is upregulated by a cAMP-dependent activation of PKA in mouse sperm. ${ }^{15}$ In the next set of experiments, we aimed to elucidate whether Cdc42-dependent modulation of CatSper function occurs upstream PKA activation.

To investigate if $\mathrm{Cdc} 42$ inhibition alters the capacitationinduced PKA activation, PKA-dependent phosphorylation was evaluated in the presence or absence of $\mathrm{Cdc} 42$ inhibitor. Incubation with MLS-573151 led to a decrease in the phosphorylation of PKA substrates (pPKAs) and tyrosine residues (pY) (Figure 7A). These data indicate that active Cdc42 is crucial for proper PKA activity. To analyze whether the requirement of $\mathrm{Cdc} 42$ is upstream PKA activation, sperm were incubated with $\mathrm{Cdc} 42$ inhibitor in the presence or absence of a membrane-permeable analog of cAMP (8-Br-cAMP) and a phosphodiesterase inhibitor (IBMX). As a result, addition of 8-Br-cAMP/IBMX bypassed the inhibition of pPKAs and pY caused by $20 \mu \mathrm{M}$ MLS-573151 (Figure 7B), suggesting that $\mathrm{Cdc} 42$ activity is required upstream PKA activation. Furthermore, the decreased in $\left[\mathrm{Ca}^{2+}\right]_{\mathrm{i}}$ provoked by MLS573151 was also bypassed by 8 -Br-cAMP/IBMX (Figure 7C and Supporting Information Figure S6A).

Since our results indicate that $\mathrm{Cdc} 42$ is acting upstream PKA activity, we analyzed whether active Cdc42 is necessary for cAMP production by sAC. Sperm cAMP levels were measured in the presence or absence of $20 \mu \mathrm{M}$ MLS-573151 and as a result, a significant decreased in cAMP concentration was observed after incubation with the $\mathrm{Cdc} 42$ inhibitor (Figure 7D). Altogether, these results suggest that Cdc42 activity is required for cAMP production by $\mathrm{sAC}$, and consequently for the upregulation of CatSper through PKA.

We also confirmed that the other Cdc42 inhibitors, that were discarded in previous experiments (Supporting Information Figure S5), display non-specific inhibition on CatSper function. Both Secramine A and CASIN provoke a decrease in phosphorylation patterns of PKAs and pY (Supporting Information Figure S6B) that could be restored by $8-\mathrm{Br}-\mathrm{cAMP} / \mathrm{IBMX}$ (Supporting Information Figure S6C), in contrast to MLS-573151, sustained $\left[\mathrm{Ca}^{2+}\right]_{\mathrm{i}}$ inhibition was not recovered even with the addition of membrane-permeable analog of cAMP and IBMX (Supporting Information Figure S6D-G). This reinforces that these drugs are not suited to study $\mathrm{Cdc} 42$ function in mouse sperm.

Since most of our results are based on only one specific Cdc42 inhibitor with no off-target effects on CatSper function (MLS-573151), two additional Cdc42 pharmacological inhibitors were also tested. ZCL278 is a small molecule inhibitor, which directly targets the binding site of intersectin, a specific Cdc42 GEF. ${ }^{65}$ ML-141 is an allosteric inhibitor that binds the guanine nucleotide-associated $\mathrm{Cdc} 42$ and induces ligand dissociation (referred to in ${ }^{66}$ as CID2950007). ZCL278 and ML-141 provoked a significant decrease in $\left[\mathrm{Ca}^{2+}\right]_{\mathrm{i}}$ (Supporting Information Figure S7A,B) and PKA activity (Supporting Information Figure S7C,D), observed as diminished phosphorylation patterns of PKAs and pY. Both effects were bypassed by the addition of 8-Br-cAMP/IBMX (Supporting Information Figure S7), suggesting that neither of these Cdc42 inhibitors presents non-specific inhibition on CatSper function. These results corroborate our observations with MLS-573151 that Cdc42 function is essential for the cAMP/PKA pathway and consequently for $\mathrm{Ca}^{2+}$ uptake through CatSper channels, and indicate that ZCL278 and ML-141 are safe to study Cdc42 on mouse sperm physiology.

Finally, to further support our approach, the CatSperdependent $\mathrm{Ca}^{2+}$ increase was investigated in the presence of PKA and CatSper inhibitors, where the addition of 8-Br-cAMP/IBMX should not be able to restore $\mathrm{Ca}^{2+}$ levels. In one case, because CatSper is stimulated downstream PKA activation and hence, direct inhibition of the kinase is not bypassed by cAMP analogs. In the other case, the direct blockade of the channel with CatSper inhibitors also impedes 

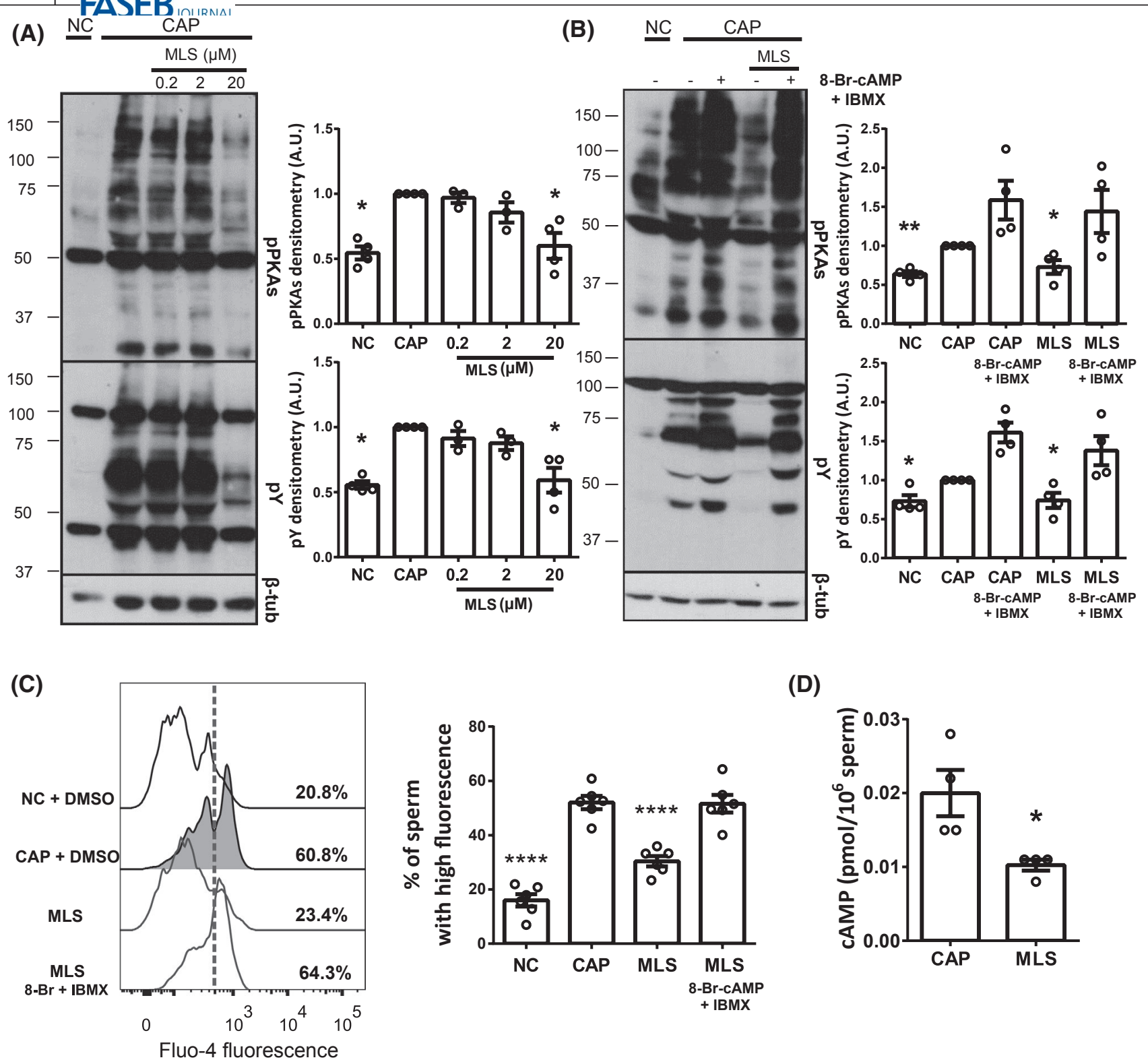

(D)
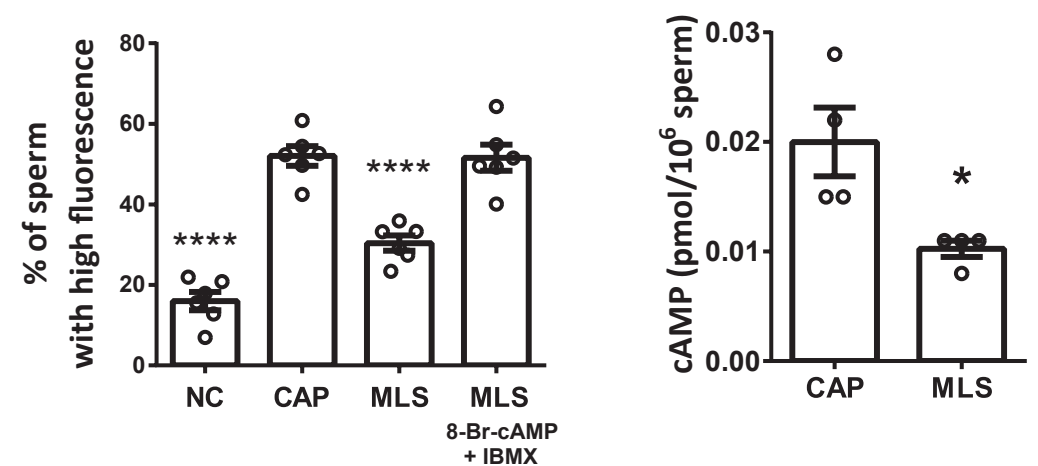

F I G U RE 7 cAMP/PKA pathway inhibition was bypassed by membrane-permeable analogs of cAMP. A and B, Protein extracts were separated by $10 \%$ SDS-PAGE and immunoblotted with antibodies against phosphotyrosine residues (pY) and substrates phosphorylated by PKA (pPKAs). As a loading control, anti- $\beta$-tubulin was used. Sperm were incubated for 90 minutes under non-capacitating (NC) and capacitating conditions (CAP) in the absence (DMSO) or presence of the appropriate inhibitor. A, Sperm were incubated with increasing amounts of MLS573151. Values represent the mean \pm SEM of at least three independent experiments, where normalization to the control condition (CAP 90 minutes with DMSO) was used. $* P<.05$ represents statistical significance vs control (CAP 90 minutes with DMSO). Non-parametric KruskalWallis test was performed in combination with Dunn's multiple comparisons test. B, Sperm were incubated under capacitating conditions with $20 \mu \mathrm{M}$ MLS-573151. The inhibition observed in this concentration was bypassed by $1 \mathrm{mM} 8$-Br-cAMP $+0.2 \mathrm{mM}$ IBMX. Values represent the mean \pm SEM of four independent experiments, where normalization to the control condition (CAP 90 minutes with DMSO) was used. $* * P<.01$; $* P<.05$ represents statistical significance vs CAP 90 minutes +8 -Br-cAMP/IBMX. Non-parametric Kruskal-Wallis test was performed in combination with Dunn's multiple comparisons test. C, $\mathrm{Ca}^{2+}$ was measured in mouse sperm incubated for 90 minutes under NC or CAP conditions in the absence (DMSO) or presence of $20 \mu \mathrm{M}$ MLS-573151. This inhibition was bypassed by $1 \mathrm{mM} 8-\mathrm{Br}-\mathrm{cAMP}+0.2 \mathrm{mM}$ IBMX. Representative histograms of normalized frequency vs Fluo-4 fluorescence of non-PI stained sperm (live), with the corresponding percentage of sperm that increased Fluo-4 fluorescence, are shown (left panel). The percentage of sperm that responds by increasing the $\left[\mathrm{Ca}^{2+}\right]_{\mathrm{i}}$ was established in the CAP control condition and extrapolated to the others conditions (dashed line). Percentage of sperm that increased Fluo-4 fluorescence after being incubated for 90 minutes in the different conditions (right panel). Values represent the mean \pm SEM of six independent experiments. $* * * * P<.0001$ represents statistical significance. One-way ANOVA with Dunnett's multiple comparisons test was performed against the control condition: CAP 90 minutes with DMSO. D, Total cAMP content of sperm incubated for 90 minutes under CAP conditions with or without $20 \mu \mathrm{M}$ MLS-573151. Values represent the mean \pm SEM of four independent experiments. $* P<.05$ represents statistical significance vs control (CAP 90 minutes with DMSO). Unpaired t test was performed 
its cAMP-dependent activation. In agreement with our interpretation of the results presented above, the decrease in the $\left[\mathrm{Ca}^{2+}\right]_{\mathrm{i}}$ promoted by either PKA inhibition (KT-5720) or CatSper blockage (NNC55-0396 or Mibefradil) was not restored by PKA activation using 8-Br-cAMP/IBMX (Supporting Information Figure S8A-C).

\section{4 | DISCUSSION}

In this report, we identified a new function of the protein Cdc42 in mouse sperm. While localizing in the CatSper signaling complex, Cdc42 activity is necessary for sAC function, and as such, it alters the observed upregulation of CatSper by cAMP-dependent pathways. SAC is canonically activated by the synergistic action of $\mathrm{HCO}_{3}{ }^{-}$and $\mathrm{Ca}^{2+}$, which modulates its enzymatic kinetics. Additionally, sAC activity also depends on the intracellular ATP concentration. Our results provide a novel molecular mechanism behind SAC regulation that helps to understand the interplay between cAMP and $\mathrm{Ca}^{2+}$ in mouse sperm. However, the mechanism by which Cdc42 modulates sAC function remains to be determined. It is possible that the protein-protein interaction between $\mathrm{Cdc} 42$ and sAC exist in sperm, but due to the lack of reliable antibodies against SAC this possibility has not been explored yet.

By super-resolution microscopy, the small GTPase Cdc 42 was localized in the sperm flagellum forming four columns along the principal piece, resembling the localization of CatSper. Additionally, CatSper1 KO sperm displayed a disorganized Cdc42 distribution in the principal piece. Previous reports demonstrate that CatSper scaffolds P-CaMKII, Caveolin-1, and PP2B in this defined quadrilateral structure, as sperm from CatSper1-null mice showed these proteins delocalized, becoming Caveolin-1 uniformly distributed in the plasma membrane. ${ }^{42}$ Therefore, our results support the idea that $\mathrm{Cdc} 42$ might be a new component of these highly organized $\mathrm{Ca}^{2+}$ domains. Until now, there is no direct evidence of these molecules regulating CatSper activity. Furthermore, Caveolin-1-null mice are fertile ${ }^{67}$ and CatSper channels in these sperm are localized normally. ${ }^{42}$ In contrast, PP2B-null mice are infertile, as their sperm cannot develop hyperactivation, which results in deficiencies to penetrate the zona pellucida. Although this evidence might suggest CatSper impairment, no defects in the principal piece were observed in PP2B-null mice, but instead these sperm exhibit a rigid mid piece. ${ }^{68}$ Interestingly, the GSK3 $\alpha$-null mice display a similar phenotype to the one observed in PP2B-null, and a recent report demonstrated that calcineurin is a phosphatase involved in the dephosphorylation of GSK3 ${ }^{69}$

Previous reports described the interaction between $\mathrm{Cdc} 42$ and Caveolin-1. Both proteins were co-immunoprecipitated from guinea pig and mouse sperm. ${ }^{3}$ However, with the exception of few reports that described the involvement of $\mathrm{Cdc} 42$ in acrosomal exocytosis, ${ }^{3,70}$ its role in mature sperm has not been conclusively demonstrated. The similar domain organization observed for both Cdc42 and Caveolin-1 may indicate possible functional interactions between these two proteins, although this needs to be further investigated.

Here we propose $\mathrm{Cdc} 42$ as a new endogenous modulator of CatSper activity in the $\mathrm{Ca}^{2+}$ signaling nanodomains orchestrated by this channel. This discovery has important implications for understanding the molecular regulation of CatSper. In this regard, recent reports found that mouse CatSper is upregulated by a cAMP-dependent activation of PKA. ${ }^{15}$ However, it is still unknown whether PKA phosphorylates one or more subunits of the channel or if its activation relies on other intermediary events. Another recent report demonstrated that mice lacking sAC do not display the increase in $\left[\mathrm{Ca}^{2+}\right]_{\mathrm{i}}$ mediated by CatSper strongly supporting the cAMP-dependent activation of this channel. ${ }^{16}$ However, other observations suggested that CatSper is not activated by cAMP/PKA signaling but is directly affected by chemicals used to probe the action of cAMP and PKA. ${ }^{62,71}$ This conclusion was obtained in experiments using human sperm and, due to the observations stated above, this would not be the case in mice. In this report, we obtain similar results in terms of CatSper function and cAMP-dependent pathways. We are proposing a new regulatory mechanism that connects CatSper function and the cAMP/PKA pathway where $\mathrm{Cdc} 42$ is important for cAMP production by $\mathrm{sAC}$, which in turn results in the activation of PKA. We hypothesize that the localization of Cdc42 in the CatSper signaling nanodomains complex is essential for the tight interplay between the increase in the $\left[\mathrm{Ca}^{2+}\right]_{\mathrm{i}}$ and the cAMP/PKA pathway. Although a cross-talk between cAMP/PKA-dependent pathways and $\mathrm{Ca}^{2+}$ plays a key role in sperm capacitation, the connection between these signaling events is incompletely understood and our results contribute to unravel this complex regulatory mechanism.

Intracellular alkalinization has been unequivocally demonstrated to alter CatSper function endogenously. ${ }^{39}$ In this regard, it was recently reported the asymmetrical distribution of the Hv1 channel in the human sperm flagellum, which is responsible, at least in part, for the increase in $\mathrm{pH}_{\mathrm{i}} \cdot{ }^{40}$ This specific localization of Hv1 could produce local alkalization when positioned in close proximity to a subset of CatSper channels resulting in asymmetric local $\mathrm{Ca}^{2+}$ changes that may be required for the complex movement of the sperm flagellum. ${ }^{72}$ In mouse, the alkalinization of the cytoplasm during capacitation occurs as a result of the sperm-specific $\mathrm{Na}^{+} / \mathrm{H}^{+}$exchanger (sNHE) $)^{73-75}$ since there is no clear evidence showing a significant role of Hv1 channels. In addition to sNHE, new reports have demonstrated the contribution in mouse sperm $\mathrm{pH}_{\mathrm{i}}$ modulation by two members of the $\mathrm{Na}^{+} /$ $\mathrm{H}^{+}$antiporter (NHA) subfamily, NHA1 and NHA2. ${ }^{16,76,77}$ Furthermore, recent reports demonstrated that EFCAB9 is a $\mathrm{pH}$ and $\mathrm{Ca}^{2+}$-dependent sensor that drives the activation 


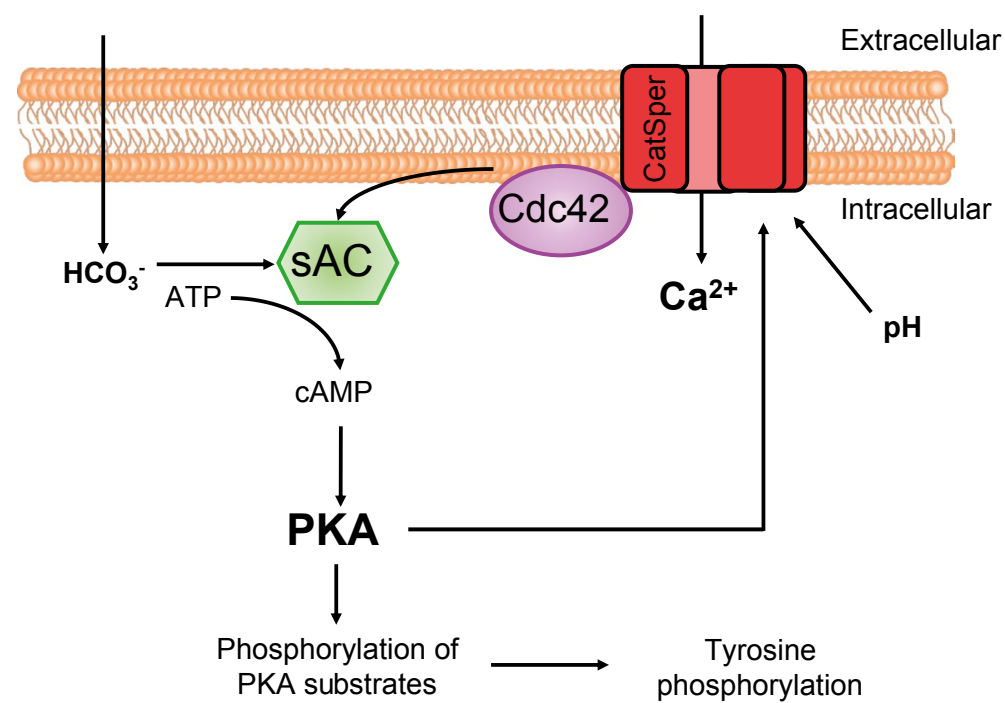

F I G U RE 8 Proposed model for CatSper activation during mouse sperm capacitation. Cdc42 is localized in the signaling complex organized by CatSper together with other proteins (not shown in this simplified model). Cdc42 is necessary for the $\mathrm{HCO}_{3}{ }^{-}$stimulation of sAC, resulting in cAMP production and activation of PKA. This activation provokes the phosphorylation of substrates (by PKA or in tyrosine residues) and the activation of CatSper (either directly or through other intermediates). As a result, a sustained $\mathrm{Ca}^{2+}$ influx promotes the development of hyperactivation

of CatSper. ${ }^{30}$ It is also possible that the highly histidinerich amino terminus of CatSper1 protein is also involved in sensing $\mathrm{pH}_{\mathrm{i}}^{21}$ since EFCAB9-null sperm display a modest CatSper activation in response to higher $\mathrm{pH}_{\mathrm{i}}{ }^{30}$ Modulation of CatSper by $\mathrm{pH}_{\mathrm{i}}$ and $\mathrm{Cdc} 42$ might be linked since a connection between $\mathrm{Cdc} 42$ and $\mathrm{H}^{+}$efflux through NHE1 has been reported in somatic cells. A bistable positive feedback regulation between $\mathrm{Cdc} 42$ and NHE1 activities has been previously proposed. ${ }^{78}$ In our experiments, the capacitation-induced $\mathrm{pH}$ alkalinization was not altered in conditions where $\mathrm{Cdc} 42$ was inhibited. However, because we only evaluated the $\mathrm{pH}_{\mathrm{i}}$ at 90 minutes of incubation, we cannot rule out that other dynamic changes in $\mathrm{pH}_{\mathrm{i}}$ might be affected at the early stages of capacitation by $\mathrm{Cdc} 42$.

Unfortunately, the global Cdc42 $\mathrm{KO}$ is embryonic lethal ${ }^{7}$ and a testis-specific conditional KO is likely to display alterations in the blood-testis barrier function, spermatocytes, and/or Sertoli cells, ${ }^{8}$ resulting in abnormal spermatogenesis. ${ }^{9}$ For this reason, we have used a pharmacological approach to study the role of Cdc42 function in mouse sperm. We are well aware that generally, inhibitors may possess off-target effects that hamper the interpretation of results. In particular, CatSper channels are sensitive to non-specific effects of multiple drugs. ${ }^{61}$ We have tried to overcome this limitation by five different specific $\mathrm{Cdc} 42$ inhibitors and so far, we have observed that two of them block CatSper in a non-specific manner (Secramine A and CASIN), while the remaining three do not (MLS-573151, ML-141, and ZCL278). In the latter case, results using these inhibitors were similar and consistent, and could be used to study Cdc42 function in mouse sperm. However, besides the extensive analysis of these compounds that we performed in our work, it is also possible that other off-target effects that we are not aware at this moment, may also exist. Thus, experiments based on pharmacological inhibitors always need to be considered with caution.

In summary, in this study, we present novel findings indicating that $\mathrm{Cdc} 42$ plays a central role in mammalian sperm capacitation. Figure 8 summarizes the results of this work in a proposed model. In the female reproductive tract (or when sperm are exposed to conditions that support capacitation), $\mathrm{HCO}_{3}{ }^{-}$stimulation of sAC resulting in cAMP production. The protein $\mathrm{Cdc} 42$ is localized in the signaling complex organized by CatSper together with other proteins (not shown in this simplified model) and its activity is required for $\mathrm{sAC}$ function. The mechanisms behind this regulation remain to be determined. The increase in cAMP levels promotes the activation of PKA which in turn, phosphorylates a subset of proteins that lead to phosphorylation in tyrosine residues of others. In addition, the stimulation of the cAMP/PKA pathway leads to the activation of CatSper (either directly or through other intermediates). As a result, a sustained $\mathrm{Ca}^{2+}$ influx promotes the development of hyperactivation. Considering that CatSper is sperm-specific and has a fundamental role in hyperactivation, this channel has emerged as a potential therapeutic target in male infertility as well as in the development of contraceptive strategies. Thus, understanding the modulation of CatSper activity and all the players involved in its regulation is of great importance.

\section{ACKNOWLEDGMENTS}

We would like to thank Drs. Gabriel Rabinovich and Mariana Salatino for their assistance in the flow cytometry experiments. We also thank Rene Baron, Fortabat, and Williams foundations. We are grateful to Dr Kirchhausen (Harvard Medical 
School, MA, USA) for providing Secramine A and Clara Marin-Briggiler, Paula Balestrini, Martina Jabloñski, and Liza Schiavi-Ehrenhaus for critically reading this manuscript.

\section{CONFLICT OF INTEREST}

The authors declare no conflict of interest.

\section{AUTHOR CONTRIBUTIONS}

G.M. Luque, X. Xu, A. Romarowski, M.G. Gervasi, G. Orta, J.L. De la Vega-Beltrán, C. Stival, and T. Dalotto-Moreno performed experiments; G.M. Luque, X. Xu, M.G. Gervasi, G. Orta, D. Krapf, and N. Gilio analyzed the data; G.M. Luque, M. G. Buffone prepared the manuscript with contributions of all other authors; A. Darszon, P.E. Visconti, and D. Krapf discussed results and contributed with ideas; G.M. Luque and M. G. Buffone designed the study.

\section{ORCID}

Guillermina M. Luque (D) https://orcid. org/0000-0002-2745-8236

María G. Gervasi (iD) https://orcid. org/0000-0002-5468-2700

Alberto Darszon (iD https://orcid.org/0000-0002-2502-0505

Mariano G. Buffone (iD https://orcid.

org/0000-0002-7163-6482

\section{REFERENCES}

1. Hall A. Rho family GTPases. Biochem Soc Trans. 2012;40(6):13781382. https://doi.org/10.1042/BST20120103.

2. Ducummon CC, Berger T. Localization of the Rho GTPases and some Rho effector proteins in the sperm of several mammalian species. Zygote. 2006;14(3):249-257. https://doi.org/10.1017/S0967 199406003790.

3. Baltiérrez-Hoyos R, Roa-Espitia AL, Hernández-González EO, Baltierrez-Hoyos R, Roa-Espitia AL, Hernandez-González EO. The association between CDC42 and caveolin-1 is involved in the regulation of capacitation and acrosome reaction of guinea pig and mouse sperm. Reproduction. 2012;144(1):123-134. https://doi. org/10.1530/REP-11-0433.

4. Romarowski A, Battistone MA, La Spina FA, et al. PKA-dependent phosphorylation of LIMK1 and Cofilin is essential for mouse sperm acrosomal exocytosis. Dev Biol. 2015;405(2):237-249. https://doi.org/10.1016/j.ydbio.2015.07.008.

5. Delgado-Buenrostro NL, Hernández-González EO, Seguranieto M, Mújica A. Actin polymerization in the equatorial and postacrosomal regions of guinea pig spermatozoa during the acrosome reaction is regulated by $\mathrm{G}$ proteins. Mol Reprod Dev. 2005;70(2):198-210. https://doi.org/10.1002/mrd.20192.

6. Fujita A, Nakamura K, Kato T, et al. Ropporin, a sperm-specific binding protein of rhophilin, that is localized in the fibrous sheath of sperm flagella. J Cell Sci. 2000;113(Pt 1):103-112.

7. Chen F, Ma L, Parrini MC, et al. Cdc42 is required for PIP(2)induced actin polymerization and early development but not for cell viability. Curr Biol. 2000;10(13):758-765.

8. Wong EWP, Mruk DD, Lee WM, Cheng CY. Regulation of bloodtestis barrier dynamics by TGF- $\beta 3$ is a Cdc 42 -dependent protein trafficking event. Proc Natl Acad Sci U S A. 2010;107(25):1139911404. https://doi.org/10.1073/pnas.1001077107.

9. Chapin RE, Wine RN, Harris MW, Borchers CH, Haseman JK. Structure and control of a cell-cell adhesion complex associated with spermiation in rat seminiferous epithelium. $J$ Androl. 2001;22(6):1030-1052. https://doi.org/10.1002/j.1939-4640.2001. tb03444.x.

10. Chang MC. Fertilizing capacity of spermatozoa deposited into the fallopian tubes. Nature. 1951;168(4277):697-698.

11. Austin CR. The capacitation of the mammalian sperm. Nature. 1952;170(4321):326. https://doi.org/10.1038/170326a0.

12. Visconti PE, Moore GD, Bailey JL, et al. Capacitation of mouse spermatozoa. II. Protein tyrosine phosphorylation and capacitation are regulated by a cAMP-dependent pathway. Development. 1995;121(4):1139-1150.

13. Alvau A, Battistone MA, Gervasi MG, et al. The tyrosine kinase FER is responsible for the capacitation-associated increase in tyrosine phosphorylation in murine sperm. Dev. 2016;143(13):23252333. https://doi.org/10.1242/dev.136499.

14. Navarrete FA, García-Vázquez FA, Alvau A, et al. Biphasic role of calcium in mouse sperm capacitation signaling pathways. $J$ Cell Physiol. 2015;230(8):1758-1769. https://doi.org/10.1002/ jcp.24873.

15. Orta G, Vega-Beltran JL, Hidalgo D, Santi CM, Visconti P, Darszon A. CatSper channels are regulated by protein kinase A. J Biol Chem. 2018;293:16830-16841. https://doi.org/10.1074/jbc. RA117.001566.

16. Balbach M, Hamzeh H, Jikeli JF, et al. Molecular mechanism underlying the action of zona-pellucida glycoproteins on mouse sperm. Front Cell Dev Biol. 2020;8. https://doi.org/10.3389/fcell.2020.572735.

17. Luque GM, Dalotto-Moreno T, Martín-Hidalgo D, et al. Only a subpopulation of mouse sperm displays a rapid increase in intracellular calcium during capacitation. J Cell Physiol. 2018;233(12):96859700. https://doi.org/10.1002/jcp.26883.

18. Suarez SS, Ho HC. Hyperactivation of mammalian sperm. Cell Mol Biol. 2003;49(3):351-356.

19. Demott RP, Suarez SS. Hyperactivated sperm progress in the mouse oviduct. Biol Reprod. 1992;46(5):779-785. https://doi. org/10.1095/biolreprod46.5.779.

20. Stauss CR, Votta TJ, Suarez SS. Sperm motility hyperactivation facilitates penetration of the hamster zona pellucida. Biol Reprod. 1995;53(6):1280-1285.

21. Ren D, Navarro B, Perez G, et al. A sperm ion channel required for sperm motility and male fertility. Nature. 2001;413(6856):603609. https://doi.org/10.1038/35098027.

22. Quill TA, Ren D, Clapham DE, Garbers DL. A voltage-gated ion channel expressed specifically in spermatozoa. Proc Natl Acad Sci U S A. 2001;98(22):12527-12531. https://doi.org/10.1073/ pnas.221454998.

23. Lobley A, Pierron V, Reynolds L, Allen L, Michalovich D. Identification of human and mouse CatSper 3 and CatSper 4 genes: Characterisation of a common interaction domain and evidence for expression in testis. Reprod Biol Endocrinol. 2003;1. https://doi. org/10.1186/1477-7827-1-53.

24. Kirichok Y, Lishko PV. Rediscovering sperm ion channels with the patch-clamp technique. Mol Hum Reprod. 2011;17(8):478-499. https://doi.org/10.1093/molehr/gar044.

25. Navarro B, Kirichok Y, Chung J-J, Clapham DE. Ion channels that control fertility in mammalian spermatozoa. Int J Dev Biol. 2008;52(5-6):607-613. https://doi.org/10.1387/ijdb.072554bn. 
26. Liu J, Xia J, Cho K-H, Clapham DE, Ren D. CatSperbeta, a novel transmembrane protein in the CatSper channel complex. $J$ Biol Chem. 2007;282(26):18945-18952. https://doi.org/10.1074/jbc. M701083200.

27. Chung J-J, Navarro B, Krapivinsky G, Krapivinsky L, Clapham DE. A novel gene required for male fertility and functional CATSPER channel formation in spermatozoa. Nat Commun. 2011;2:153. https://doi.org/10.1038/ncomms1153.

28. Wang H, Liu J, Cho K-H, Ren D. A novel, single, transmembrane protein CATSPERG is associated with CATSPER1 channel protein. Biol Reprod. 2009;81(3):539-544. https://doi.org/10.1095/ biolreprod.109.077107.

29. Chung J-J, Miki K, Kim D, et al. CatSperל regulates the structural continuity of sperm $\mathrm{Ca} 2+$ signaling domains and is required for normal fertility. eLife. 2017;6:e23082. https://doi.org/10.7554/ eLife. 23082.

30. Hwang JY, Mannowetz N, Zhang Y, et al. Dual sensing of physiologic $\mathrm{pH}$ and calcium by EFCAB9 regulates sperm motility. Cell. 2019;177(6):1480-1494.e19. https://doi.org/10.1016/j. cell.2019.03.047.

31. Qi H, Moran MM, Navarro B, et al. All four CatSper ion channel proteins are required for male fertility and sperm cell hyperactivated motility. Proc Natl Acad Sci U S A. 2007;104(4):1219-1223. https://doi.org/10.1073/pnas.0610286104.

32. Quill TA, Sugden SA, Rossi KL, Doolittle LK, Hammer RE, Garbers DL. Hyperactivated sperm motility driven by CatSper2 is required for fertilization. Proc Natl Acad Sci U S A. 2003;100(25):1486914874. https://doi.org/10.1073/pnas.2136654100.

33. Avenarius MR, Hildebrand MS, Zhang Y, et al. Human male infertility caused by mutations in the CATSPER1 channel protein. Am J Hum Genet. 2009;84(4):505-510. https://doi.org/10.1016/j. ajhg.2009.03.004.

34. Avidan N, Tamary H, Dgany O, et al. CATSPER2, a human autosomal nonsyndromic male infertility gene. Eur J Hum Genet. 2003;11(7):497-502. https://doi.org/10.1038/sj.ejhg.5200991.

35. Smith JF, Syritsyna O, Fellous M, et al. Disruption of the principal, progesterone-activated sperm $\mathrm{Ca} 2+$ channel in a CatSper2-deficient infertile patient. Proc Natl Acad Sci U S A. 2013;110(17):68236828. https://doi.org/10.1073/pnas.1216588110.

36. Lishko PV, Botchkina IL, Kirichok Y. Progesterone activates the principal $\mathrm{Ca} 2+$ channel of human sperm. Nature. 2011;471(7338):387-391. https://doi.org/10.1038/nature09767.

37. Miller MR, Mansell SA, Meyers SA, Lishko PV. Flagellar ion channels of sperm: similarities and differences between species. Cell Calcium. 2015;58(1):105-113. https://doi.org/10.1016/j. ceca.2014.10.009.

38. Miller MR, Mannowetz N, Iavarone AT, et al. Unconventional endocannabinoid signaling governs sperm activation via the sex hormone progesterone. Science. 2016;352(6285):555-559. https://doi. org/10.1126/science.aad6887.

39. Kirichok Y, Navarro B, Clapham DE. Whole-cell patch-clamp measurements of spermatozoa reveal an alkaline-activated $\mathrm{Ca} 2+$ channel. Nature. 2006;439:737-740. https://doi.org/10.1038/natur e04417.

40. Lishko PV, Botchkina IL, Fedorenko A, Kirichok Y. Acid extrusion from human spermatozoa is mediated by flagellar voltagegated proton channel. Cell. 2010;140(3):327-337. https://doi. org/10.1016/j.cell.2009.12.053.
41. Lishko PV, Mannowetz N. CatSper: a unique calcium channel of the sperm flagellum. Curr Opin Physiol. 2018;2:109-113. https:// doi.org/10.1016/j.cophys.2018.02.004.

42. Chung J-JJ, Shim S-HH, Everley RA, Gygi SP, Zhuang X, Clapham DE. Structurally distinct $\mathrm{Ca} 2+$ signaling domains of sperm flagella orchestrate tyrosine phosphorylation and motility. Cell. 2014;157(4):808-822. https://doi.org/10.1016/j.cell.2014.02.056.

43. Navarrete FA, Alvau A, Lee HC, et al. Transient exposure to calcium ionophore enables in vitro fertilization in sterile mouse models. Sci Rep. 2016;6:33589. https://doi.org/10.1038/srep33589.

44. Weigel AV, Simon B, Tamkun MM, Krapf D. Ergodic and nonergodic processes coexist in the plasma membrane as observed by single-molecule tracking. Proc Natl Acad Sci. 2011;108(16):64386443. https://doi.org/10.1073/pnas.1016325108.

45. Campagnola G, Nepal K, Schroder BW, Peersen OB, Krapf D. Superdiffusive motion of membrane-targeting C2 domains. Sci Rep. 2015;5(1):17721. https://doi.org/10.1038/srep17721.

46. Izeddin I, El Beheiry M, Andilla J, Ciepielewski D, Darzacq X, Dahan M. PSF shaping using adaptive optics for three-dimensional single-molecule super-resolution imaging and tracking. Opt Express. 2012;20(5):4957. https://doi.org/10.1364/OE.20.004957.

47. Gervasi MG, Xu X, Carbajal-Gonzalez B, Buffone MG, Visconti PE, Krapf D. The actin cytoskeleton of the mouse sperm flagellum is organized in a helical structure. J Cell Sci. 2018;131:jcs.215897. https://doi.org/10.1242/jcs.215897.

48. Huang B, Wang W, Bates M, Zhuang X. Three-dimensional superresolution imaging by stochastic optical reconstruction microscopy. Science. 2008;319(5864):810-813. https://doi.org/10.1126/ science. 1153529.

49. Ovesný M, Kř́žžek P, Borkovec J, Švindrych Z, Hagen GM. ThunderSTORM: a comprehensive ImageJ plug-in for PALM and STORM data analysis and super-resolution imaging. Bioinformatics. 2014;30(16):2389-2390. https://doi.org/10.1093/ bioinformatics/btu202.

50. Stival C, Ritagliati C, Xu X, et al. Disruption of protein kinase A localization induces acrosomal exocytosis in capacitated mouse sperm. J Biol Chem. 2018;293(24):jbc.RA118.002286. https://doi. org/10.1074/jbc.RA118.002286.

51. Escoffier J, Krapf D, Navarrete F, Darszon A, Visconti PE. Flow cytometry analysis reveals a decrease in intracellular sodium during sperm capacitation. J Cell Sci. 2012;125(2):473-485. https://doi. org/10.1242/jcs.093344.

52. Demarco IA, Espinosa F, Edwards J, et al. Involvement of a $\mathrm{Na}+/$ HCO3-cotransporter in mouse sperm capacitation. J Biol Chem. 2003;278(9):7001-7009. https://doi.org/10.1074/jbc.M206284200.

53. López-González I, Torres-Rodríguez P, Sánchez-Carranza O, et al. Membrane hyperpolarization during human sperm capacitation. Mol Hum Reprod. 2014;20(7):619-629. https://doi.org/10.1093/ molehr/gau029.

54. Ernesto JI, Weigel Muñoz M, Battistone MA, et al. CRISP1 as a novel CatSper regulator that modulates sperm motility and orientation during fertilization. J Cell Biol. 2015;210(7):1213-1224. https://doi.org/10.1083/jcb.201412041.

55. Rust MJ, Bates M, Zhuang X. STORM. Nat Meth. 2006;3(10):793796. https://doi.org/10.1038/nmeth929.

56. Rossman KL, Der CJ, Sondek J. GEF means go: turning on RHO GTPases with guanine nucleotide-exchange factors. Nat Rev Mol Cell Biol. 2005;6(2):167-180. https://doi.org/10.1038/nrm1587. 
57. Garcia-Mata R, Boulter E, Burridge K. The, "invisible hand": regulation of RHO GTPases by RHOGDIs. Nat Rev Mol Cell Biol. 2011;12(8):493-504. https://doi.org/10.1038/nrm3153.

58. Surviladze Z, Waller A, Wu Y, et al. Identification of a small GTPase inhibitor using a high-throughput flow cytometry beadbased multiplex assay. J Biomol Screen. 2010;15(1):10-20. https:// doi.org/10.1177/1087057109352240.

59. Espinosa F, Darszon A. Mouse sperm membrane potential: changes induced by Ca2+. FEBS Lett. 1995;372:119-125.

60. Torres-Flores V, Picazo-Juárez G, Hernández-Rueda Y, Darszon A, González-Martínez MT. Sodium influx induced by external calcium chelation decreases human sperm motility. Hum Reprod. 2011;26(10):2626-2635. https://doi.org/10.1093/humrep/der237.

61. Barratt CL, Publicover SJ. Sperm are promiscuous and CatSper is to blame.... EMBO J. 2012;31(7):1624-1626. https://doi. org/10.1038/emboj.2012.62.

62. Brenker C, Goodwin N, Weyand I, et al. The CatSper channel: a polymodal chemosensor in human sperm. EMBO J. 2012;31(7):1654-1665. https://doi.org/10.1038/emboj.2012.30.

63. Pelish HE, Peterson JR, Salvarezza SB, et al. Secramine inhibits Cdc42-dependent functions in cells and $\mathrm{Cdc} 42$ activation in vitro. Nat Chem Biol. 2006;2(1):39-46. https://doi.org/10.1038/nchem bio751.

64. Peterson JR, Lebensohn AM, Pelish HE, Kirschner MW. Biochemical suppression of small-molecule inhibitors: a strategy to identify inhibitor targets and signaling pathway components. Chem Biol. 2006;13(4):443-452. https://doi.org/10.1016/j.chemb iol.2006.02.009.

65. Friesland A, Zhao Y, Chen YH, Wang L, Zhou H, Lu Q. Small molecule targeting Cdc42-intersectin interaction disrupts Golgi organization and suppresses cell motility. Proc Natl Acad Sci U S A. 2013;110(4):1261-1266. https://doi.org/10.1073/pnas.11160 51110.

66. Hong L, Kenney SRay, Phillips GK, et al. Characterization of a Cdc42 protein inhibitor and its use as a molecular probe. J Biol Chem. 2013;288(12):8531-8543. https://doi.org/10.1074/jbc. M112.435941.

67. Razani B, Engelman JA, Wang XB, et al. Caveolin-1 null mice are viable but show evidence of hyperproliferative and vascular abnormalities. J Biol Chem. 2001;276(41):38121-38138. https://doi. org/10.1074/jbc.M105408200.

68. Miyata H, Satouh Y, Mashiko D, et al. Sperm calcineurin inhibition prevents mouse fertility with implications for male contraceptive. Science. 2015;350(6259):442-445. https://doi.org/10.1126/ science.aad0836.

69. Dey S, Eisa A, Kline D, Wagner FF, Abeysirigunawardena S, Vijayaraghavan S. Roles of glycogen synthase kinase 3 alpha and calcineurin in regulating the ability of sperm to fertilize eggs. FASEB J. 2020;34(1):1247-1269. https://doi.org/10.1096/fj.201902163R.

70. Angeles-Floriano T, Roa-Espitia AL, Baltiérrez-Hoyos R, CorderoMartínez J, Elizondo G, Hernández-González EO. Absence of aryl hydrocarbon receptor alters CDC42 expression and prevents actin polymerization during capacitation. Mol Reprod Dev. 2016;83(11):1015-1026. https://doi.org/10.1002/mrd.22736.

71. Wang T, Young S, Krenz H, et al. The $\mathrm{Ca} 2+$ channel CatSper is not activated by cAMP/PKA signaling but directly affected by chemicals used to probe the action of cAMP and PKA. J Biol Chem. 2020;295(38):13181-13193. https://doi.org/10.1074/jbc. RA120.013218.

72. Miller MR, Kenny SJ, Mannowetz N, et al. Asymmetrically positioned flagellar control units regulate human sperm rotation. Cell Rep. 2018;24(10):2606-2613. https://doi.org/10.1016/j. celrep.2018.08.016.

73. Chávez JC, Ferreira JJ, Butler A, et al. SLO3 K+ channels control calcium entry through CATSPER channels in sperm. $J$ Biol Chem. 2014;289(46):32266-32275. https://doi.org/10.1074/jbc. M114.607556.

74. Wang XF, Zhou CX, Shi QX, et al. Involvement of CFTR in uterine bicarbonate secretion and the fertilizing capacity of sperm. Nat Cell Biol. 2003;5(10):902-906. https://doi.org/10.1038/ncb1047.

75. Wang D, Hu J, Bobulescu IA, et al. A sperm-specific $\mathrm{Na}+\mathrm{H}+$ exchanger (sNHE) is critical for expression and in vivo bicarbonate regulation of the soluble adenylyl cyclase (sAC). Proc Natl Acad Sci. 2007;104(22):9325-9330. https://doi.org/10.1073/pnas.0611296104.

76. Liu T, Huang J-C, Lu C-L, et al. Immunization with a DNA vaccine of testis-specific sodium-hydrogen exchanger by oral feeding or nasal instillation reduces fertility in female mice. Fertil Steril. 2010;93(5):1556-1566. https://doi.org/10.1016/j.fertn stert.2009.03.056.

77. Chen S-R, Chen M, Deng S-L, Hao X-X, Wang X-X, Liu Y-X. Sodium-hydrogen exchanger NHA1 and NHA2 control sperm motility and male fertility. Cell Death Dis. 2016;7(3):e2152. https:// doi.org/10.1038/cddis.2016.65.

78. Frantz C, Karydis A, Nalbant P, Hahn KM, Barber DL. Positive feedback between $\mathrm{Cdc} 42$ activity and $\mathrm{H}^{+}$efflux by the $\mathrm{Na}-\mathrm{H}$ exchanger NHE1 for polarity of migrating cells. J Cell Biol. 2007;179(3):403-410. https://doi.org/10.1083/jcb.200704169.

\section{SUPPORTING INFORMATION}

Additional Supporting Information may be found online in the Supporting Information section.

How to cite this article: Luque GM, Xu X, Romarowski A, et al. Cdc42 localized in the CatSper signaling complex regulates cAMP-dependent pathways in mouse sperm. The FASEB Journal. 2021;35:e21723. https://doi.org/10.1096/fj.20200 2773RR 